ARTICLE

\title{
The mechanosensitive Piezo1 channel mediates heart mechano-chemo transduction
}

\author{
Fan Jiang ${ }^{1}$, Kunlun Yin², Kun Wu ${ }^{1,5}$, Mingmin Zhang ${ }^{1}$, Shiqiang Wang ${ }^{3}$, Heping Cheng ${ }^{4}{ }^{4}$ Zhou Zhou ${ }^{2} \&$ \\ Bailong Xiao (1) ${ }^{1 \times}$
}

The beating heart possesses the intrinsic ability to adapt cardiac output to changes in mechanical load. The century-old Frank-Starling law and Anrep effect have documented that stretching the heart during diastolic filling increases its contractile force. However, the molecular mechanotransduction mechanism and its impact on cardiac health and disease remain elusive. Here we show that the mechanically activated Piezo1 channel converts mechanical stretch of cardiomyocytes into $\mathrm{Ca}^{2+}$ and reactive oxygen species (ROS) signaling, which critically determines the mechanical activity of the heart. Either cardiac-specific knockout or overexpression of Piezo1 in mice results in defective $\mathrm{Ca}^{2+}$ and ROS signaling and the development of cardiomyopathy, demonstrating a homeostatic role of Piezo1. Piezo1 is pathologically upregulated in both mouse and human diseased hearts via an autonomic response of cardiomyocytes. Thus, Piezol serves as a key cardiac mechanotransducer for initiating mechano-chemo transduction and consequently maintaining normal heart function, and might represent a novel therapeutic target for treating human heart diseases.

\footnotetext{
${ }^{1}$ State Key Laboratory of Membrane Biology, Tsinghua-Peking Center for Life Sciences, Beijing Advanced Innovation Center for Structural Biology, IDG/ McGovern Institute for Brain Research, School of Pharmaceutical Sciences, Tsinghua University, Beijing 100084, China. ${ }^{2}$ State Key Laboratory of Cardiovascular Disease, Beijing Key Laboratory for Molecular Diagnostics of Cardiovascular Diseases, Center of Laboratory Medicine, Fuwai Hospital, National Center for Cardiovascular Diseases, Chinese Academy of Medical Sciences and Peking Union Medical College, Beijing 100037, China. ${ }^{3}$ State Key Laboratory of Membrane Biology, College of Life Sciences and Institute of Molecular Medicine, Peking University, Beijing, China. ${ }^{4}$ State Key Laboratory of Membrane Biology, Institute of Molecular Medicine, Peking-Tsinghua Center for Life Sciences, Peking University, Beijing, China. ${ }^{5}$ Present address: Medical Research Center, Beijing Key Laboratory of Cardiopulmonary Cerebral Resuscitation, Department of Emergency, Beijing Chao-Yang Hospital, Capital Medical University, Beijing, China. ${ }^{凶}$ email: xbailong@mail.tsinghua.edu.cn
} 
eart experiences drastic mechanical changes on a beat-tobeat basis and evolves intrinsic mechanisms to adapt cardiac output to hemodynamic conditions ${ }^{1}$. Stretching the ventricular wall due to an increase in end-diastolic volume leads to an immediate enhancement of cardiac contraction, followed by a slow response lasting for minutes. These responses in the intact heart have been respectively described as the Frank-Starling law and the Anrep effect for over 100 years $^{2-4}$, which constitute powerful mechanisms to allow the heart to adapt to an abrupt rise in either preload or afterload. While the Frank-Starling phenomenon is in part due to a sensitization of the myofibrillar $\mathrm{Ca}^{2+}$ sensitivity ${ }^{5,6}$, an increase in intracellular $\mathrm{Ca}^{2+}$ might play a key role in mediating the stretch-induced biphasic enhancement of cardiac contraction ${ }^{7,8}$. It has been proposed that stretch-activated cation channels might provide a conceptually simple mechanism for conferring cardiac mechanosensitivity and the resulting $\mathrm{Ca}^{2+}$ signaling 9,10, which determines the strength of cardiac contraction ${ }^{11}$. However, the molecular identify of the underlying mechanotransduction channel and its role in normal and diseased hearts have remained unclear.

Piezol is a bona fide mechanosensitive cation channel ${ }^{12-15}$ that mediates mechanically activated cationic currents and $\mathrm{Ca}^{2+}$ signaling in various cell types ${ }^{16-19}$, including endothelial cells, red blood cells, smooth muscle cells, epithelial cells, osteoblasts, and cardiac fibroblasts ${ }^{20-26}$. Piezol is involved in various aspects of vascular physiology 27 , including blood and lymphatic vessel development ${ }^{20,21,28-31}$, vascular tone $\mathrm{e}^{23,32}$, arterial remodeling 22 , and red blood cell volume homeostasis $^{24}$. Furthermore, together with its homolog member Piezo2, Piezo1 has been proposed as the mechanosensor in baroreceptor neurons for baroreflex control of blood pressure and heart rate $\mathrm{e}^{33}$. However, the expression and role of Piezol in cardiomyocytes and heart function have not been genetically studied.

Here, we use mouse genetics and pharmacology to demonstrate the critical role of Piezol in cardiac mechano-chemo transduction in normal and diseased hearts.

\section{Results}

Expression and localization of Piezol proteins in cardiomyocytes. Previous studies have reported relatively low level of Piezo1 mRNA expression in heart tissues and primarily cultured cardiomyocytes $^{12,26,34}$. However, the abundance and localization of Piezol proteins in cardiomyocytes have not been systematically characterized. To enable detection of Piezol proteins expressed in mouse hearts, we used the CRISPR/Cas9 technology to generate a Piezo1-Flag-knock-in mouse line (Piezol-Flag-KI) (Supplementary Fig. 1a, b), in which the Flag-coding sequence was inserted into the genetic sequence coding the mouse Piezo1 (Supplementary Fig. 1b). Importantly, the Flag-tagged Piezo1 (Piezo1Flag) proteins were specifically detected in the Piezol-Flag-KI heart tissue either via western blotting of the anti-Flagimmunoprecipitated sample (Supplementary Fig. 1c) or via immunostaining with the anti-Flag antibody (Supplementary Fig. 1d). As a control, heart tissues derived from the wild-type littermate control mice showed no Piezol-Flag expression (Supplementary Fig. 1c, d). Interestingly, immunostaining of primarily cultured adult ventricular myocytes using the anti-Flag antibody revealed punctate and striated expression pattern of the Piezo1Flag protein specifically in the Piezo1-Flag-KI cells, but not in the control cells (Fig. 1a), which was similar to the expression pattern of the Z-line marker protein actinin (Fig. 1a), indicating the localization of Piezol near the T-tubule. The endogenous expression of Piezol in cardiomyocytes was further confirmed using the previously reported Piezol-tdTomato knock-in mouse line (Piezo1-tdTomato-KI) (Fig. 1b), in which the tdTomato coding sequence was knocked into the genetic sequence coding the C-terminus of the mouse Piezol protein ${ }^{20}$. Immunostaining using the anti-dsRed antibody clearly detected the expression of the Piezo1-tdTomato proteins in the Piezo1-tdTomato-KI cardiomyocytes, but not in the control cells (Fig. 1b). We have previously found that Piezol localized in the plasma membrane interacts with the Sarco/Endoplasmic-Reticulum Calcium ATPase $(\mathrm{SERCA})^{35}$, which plays a key role in recycling cytosolic $\mathrm{Ca}^{2+}$ back into the sarcoplasmic reticulum (SR) for maintaining SR $\mathrm{Ca}^{2+}$ content of cardiomyocytes ${ }^{36}$. Indeed, co-immunostaining of
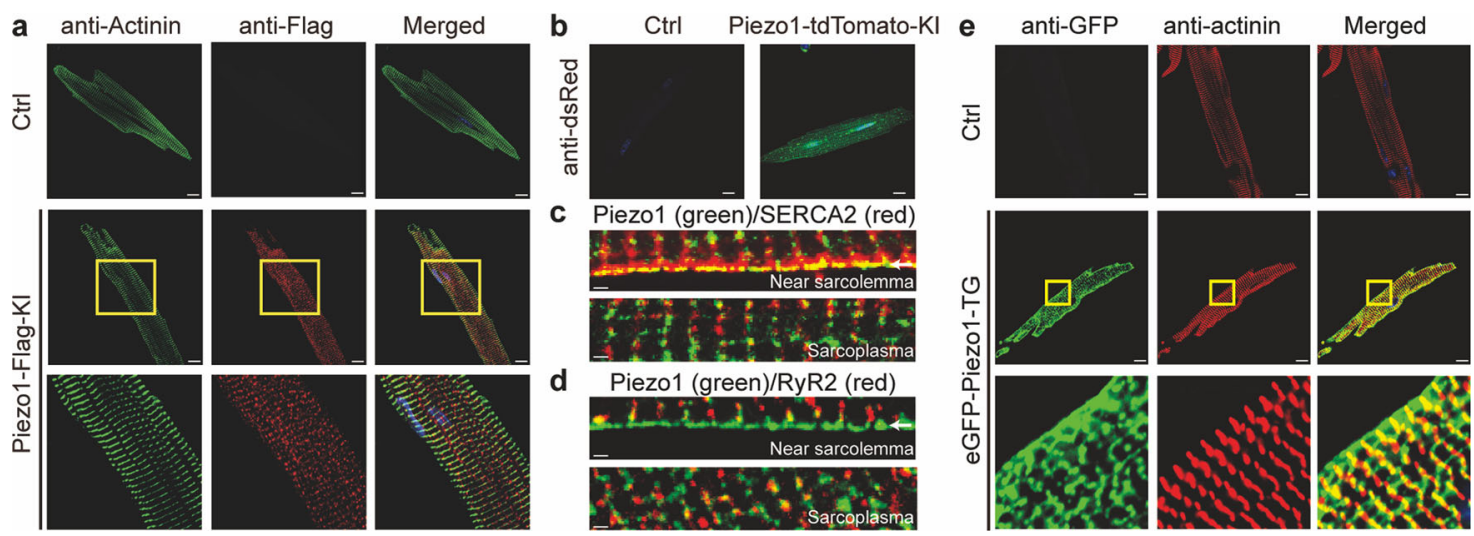

Fig. 1 Expression and localization of Piezo1 in mouse cardiomyocytes. a Immunofluorescent staining of adult cardiomyocytes isolated from either the control littermates (Ctrl) or Piezo1-Flag-KI mice. Green shows the anti- $\alpha$-actinin signal for displaying the Z-lines of the striated cardiomyocytes, while red shows the anti-Flag signal for the endogenously expressed Flag-tagged Piezo1 protein. The bottom panel shows the enlarged section marked with the yellow box in the middle panel. Scale bar, $10 \mu \mathrm{m}$. b Immunofluorescent staining of adult cardiomyocytes isolated from either the control littermates (Ctrl) or the Piezo1-tdTomato-KI mice with the anti-dsRed antibody. Scale bar, $10 \mu \mathrm{m}$. c, d Co-immunofluorescent staining of the endogenously expressed Piezo1 with either SERCA2 (c) or RyR2 (d) in cardiomyocytes derived from the Piezo1-tdTomato-KI mice. The near plasma membrane and sarcoplasmic regions are respectively shown in the top and bottom panels. The Piezo1 signal is shown in green, while the signal of SERCA2 or RyR2 in red. The white arrow on the top panel indicates the sarcolemma region. e Immunofluorescent staining of adult cardiomyocytes isolated from either the control littermates (Ctrl) or the eGFP-Piezo1-TG mice. Green shows the anti-GFP signal for displaying the cardiac specific overexpression of the eGFP-Piezo1 fusion proteins, while red shows the anti- $\alpha$-actinin signal. The bottom panel shows the enlarged section marked with the yellow box in the middle panel. Scale bar, $10 \mu \mathrm{m}$. Each experiment was repeated independently three times with similar results. 
the endogenously expressed Piezo1-tdTomato and the type 2 SERCA (SERCA2) in cardiomyocytes revealed their colocalization near the sarcolemma but not inside the sarcoplasma (Fig. 1c). In contrast, sarcolemma-localized Piezo1 was not co-localized with the SR-localized type 2 ryanodine receptor (RyR2) (Fig. 1d), which is the intracellular $\mathrm{Ca}^{2+}$ release channel responsible for the elementary $\mathrm{Ca}^{2+}$ release, the so-called $\mathrm{Ca}^{2+}$ spark, which underlies the cardiac excitation-contraction coupling 37,38 . Together, these data demonstrate that endogenous Piezol proteins are clearly expressed in the sarcolemma of adult cardiomyocytes.

We also generated cardiac-specific transgenic mice overexpressing the eGFP-Piezol fusion protein by crossing the MLC2v (myosin light chain $2 \mathrm{v}$ )-Cre mice that exclusively express the Cre recombinase in ventricular cardiomyocytes ${ }^{39}$ with the

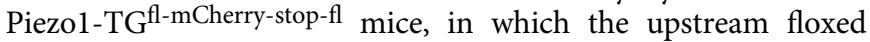
mCherry coding sequence with a stop codon blocks the translation of the downstream eGFP-Piezol fusion protein 40 (Supplementary Fig. 2b). The resulting Piezo1-TG ${ }^{\text {fl-mCherry-stop-fl/ }}$ MLC2v-Cre (Piezo1-TG) mice had the floxed mCherry-stop codon sequence excised and accordingly expressed the eGFPPiezol fusion proteins. Notably, the overexpressed eGFP-Piezo1 proteins showed similar punctate and striated localization patterns as that of the endogenously expressed Piezo1 (Fig. 1e).

Piezo1 mediates Yodal induced $\mathrm{Ca}^{2+}$ responses in cardiomyocytes. To assay the functional role of the cardiac Piezo1, we generated cardiac-specific Piezol-deficient mice (KO) by crossing the Piezo ${ }^{\mathrm{fl} / \mathrm{fl}}$ mice $^{24}$ with the MLC2v-Cre mice ${ }^{39}$ (Supplementary Fig. 2a). RT-PCR and western blotting confirmed the successful deletion of Piezol in the KO heart (Fig. 2a, b). In contrast, the expression of Piezo1 in lung, blood vessel, and red blood cells was not affected (Fig. 2a and Supplementary Fig. 2c), suggesting specific deletion of Piezol in cardiomyocytes. Given that it is highly challenging to record stretch-activated currents from adult cardiomocytes $^{41}$, we have employed single-cell $\mathrm{Ca}^{2+}$ imaging to functionally assay Piezol activity in cardiomyocytes in response to Yoda1, which is a chemical activator of Piezol with an apparent $\mathrm{EC}_{50}$ (the concentration for causing half maximal activation) of $\sim 17 \mu \mathrm{M}$ and a maximal water solubility of $\sim 30 \mu \mathrm{M}^{42}$. We thus used $30 \mu \mathrm{M}$ Yodal for maximal activation of endogenously expressed Piezol in cardiomyocytes. For comparison, we used $10 \mathrm{mM}$ caffeine to activate RyR2 for measuring the total SR $\mathrm{Ca}^{2+}$ content, which is a key determinant of cardiac $\mathrm{Ca}^{2+}$ signaling and excitation-contraction coupling ${ }^{11}$. Yodal induced a sustained $\mathrm{Ca}^{2+}$ response in cardiomyocytes derived from the littermate control mice in the presence of $1.8 \mathrm{mM}$ extracellular $\mathrm{Ca}^{2+}$ (Fig. 2c, d). Removing the extracellular $\mathrm{Ca}^{2+}$ abolished the response, suggesting Yodal-induced $\mathrm{Ca}^{2+}$ influx (Fig. 2e). The amplitude of the $\mathrm{Ca}^{2+}$ increase reached about $94 \%$ of the caffeine response (Fig. 2c, d, f, g), suggesting relatively robust Yoda1induced $\mathrm{Ca}^{2+}$ increase in cardiomyocytes. Remarkably, the Yodal response was nearly completely abolished in cardiomyocytes derived from the Piezo1-KO mice (Fig. 2c, d). On the other hand, cardiomyocytes derived from the Piezol-TG mice had significantly larger Yoda1-induced $\mathrm{Ca}^{2+}$ response than that detected in the littermate control cells (Fig. 2j, k). Unexpectedly, the caffeine-induced $\mathrm{Ca}^{2+}$ release was significantly reduced in both Piezo1-KO (Fig. 2f, g) and Piezo1-TG (Fig. 2l) cardiomyocytes compared to their respective control cells, indicating decreased SR $\mathrm{Ca}^{2+}$ content. To exclude the possibility that the diminished Yoda1-response in the $\mathrm{KO}$ cells might be due to their decreased $\mathrm{SR} \mathrm{Ca}{ }^{2+}$ content, which might result in impaired $\mathrm{Ca}^{2+}$-induced $\mathrm{Ca}^{2+}$ release (CICR), we carried out total internal reflection fluorescent microscopy to image localized $\mathrm{Ca}^{2+}$ influx near the plasma membrane. Remarkably, we detected rapid and robust
Yoda1-induced $\mathrm{Ca}^{2+}$ events in control cardiomyocytes, which were nearly abolished in the KO cells (Fig. 2h, i). These data collectively demonstrate that Piezol mediates Yoda1-induced $\mathrm{Ca}^{2+}$ responses in cardiomyocytes.

Notably, compared to caffeine-induced $\mathrm{Ca}^{2+}$ response (Fig. 2f), the Yoda1-induced response in both control and Piezo1-TG cells appeared to be slower, but more sustained (Fig. $2 c, j$ ). These data suggest that the relatively slow global increase of cytosolic $\mathrm{Ca}^{2+}$ evoked by Yoda1 was not caused by the expression level of Piezo1. Instead, we noticed that the onset of Yoda1-induced localized $\mathrm{Ca}^{2+}$ influx (Fig. $2 \mathrm{~h}$ ) appeared to be much faster than Yoda1-induced global cytosolic $\mathrm{Ca}^{2+}$ increase assayed by singlecell $\mathrm{Ca}^{2+}$ imaging (Fig. 2c, j). Thus, we reasoned that the Yoda1induced global cytosolic $\mathrm{Ca}^{2+}$ increase might result from a summation of unsynchronized $\mathrm{Ca}^{2+}$ influx mediated by Piezol distributed in the large sarcolemma area including T-tubules (Fig. 1), resulting in the relatively slow onset of the Yoda1 response observed in single-cell $\mathrm{Ca}^{2+}$ imaging.

Piezol mediates stretch-induced $\mathrm{Ca}^{2+}$ sparks in cardiomyocytes. Previous studies have shown that an $8 \%$ of diastolic stretch of ventricular myocytes can lead to a burst of $\mathrm{Ca}^{2+}$ sparks ${ }^{43,44}$. To examine whether Piezol might mediate such stretch-induced $\mathrm{Ca}^{2+}$ response, we have adopted the previously developed MyoTak system to precisely stretch a single cardiomyocyte and simultaneously measured the occurrence of $\mathrm{Ca}^{2+}$ sparks using line-scan confocal microscopy ${ }^{43}$. Consistent with previous reports ${ }^{43,44}$, an $8 \%$ stretch of the control cardiomyocytes evoked an apparent and rapid increase in $\mathrm{Ca}^{2+}$ sparks, which was not observed in the Piezo1-KO cardiomyocytes (Fig. 3a, b). The fold change of $\mathrm{Ca}^{2+}$ spark rate upon stretch in the control cardiomyocytes $(7.2 \pm 2.7$ folds) is significantly higher than that observed in the KO cardiomyocytes ( $1.4 \pm 0.2$ folds) (Fig. $3 \mathrm{c})$. The stretch-induced $\mathrm{Ca}^{2+}$ sparks were largely recovered upon relaxation from the stretch (Fig. 3c). To further demonstrate stretch dependence of $\mathrm{Ca}^{2+}$ sparks, we applied a $15 \%$ of stretch that is within the range of a diastolic stretch normally occurred during a healthy heart beat. Compared to the $8 \%$ stretch-induced response, $15 \%$ stretch induced a relatively more robust increase in $\mathrm{Ca}^{2+}$ sparks, which could still largely recover upon relaxation (Fig. 3d, e), indicating no major damage to the cells. Importantly, such responses were not observed in the Piezo1-KO cardiomyocytes (Fig. 3d, e). To exclude the possibility that the lack of stretch-induced $\mathrm{Ca}^{2+}$ spark in the KO cells might be due to their decreased $\mathrm{SR} \mathrm{Ca}^{2+}$ content, we employed $1 \mathrm{mM}$ caffeine to trigger $\mathrm{Ca}^{2+}$ sparks (Fig. 3f, g). Despite relatively lower $\mathrm{Ca}^{2+}$ spark events in the KO cardiomyocytes due to their reduced SR $\mathrm{Ca}^{2+}$ content, the caffeine-induced fold change of $\mathrm{Ca}^{2+}$ sparks were similar between the control and KO cells (Fig. 3g). These data suggest that the Piezo1-KO cardiomyocytes specifically lost stretch-induced but retained caffeine-induced $\mathrm{Ca}^{2+}$ sparks. Taken together, these data demonstrate that Piezol mediates stretchinduced $\mathrm{Ca}^{2+}$ sparks in cardiomyocytes.

Piezol affects $\mathrm{Ca}^{2+}$ homeostasis in cardiomyocytes. The observation that Piezo1-KO cardiomyocytes had both reduced SR $\mathrm{Ca}^{2+}$ content and less spontaneous $\mathrm{Ca}^{2+}$ sparks prior to stretch stimulation has prompted us to further examine the role of Piezol in controlling cardiac $\mathrm{Ca}^{2+}$ homeostasis. Indeed, a direct comparison of the spontaneous $\mathrm{Ca}^{2+}$ sparks between the control and KO cardiomyocytes revealed significantly less frequent $\mathrm{Ca}^{2+}$ spark events in the $\mathrm{KO}$ cells than in the control cells $(0.5 \pm 0.6$ vs $2.0 \pm 0.2$ events per $10 \mu \mathrm{m}$ per second, respectively) (Fig. 4a, c). On the basis of histogram analysis, the peak amplitude of the $\mathrm{Ca}^{2+}$ spark from the KO cells was slightly lower than that from 
a

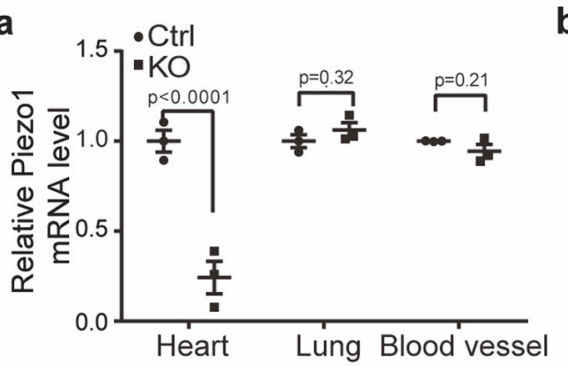

c

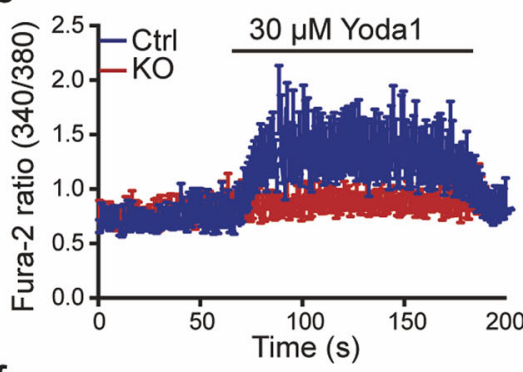

f

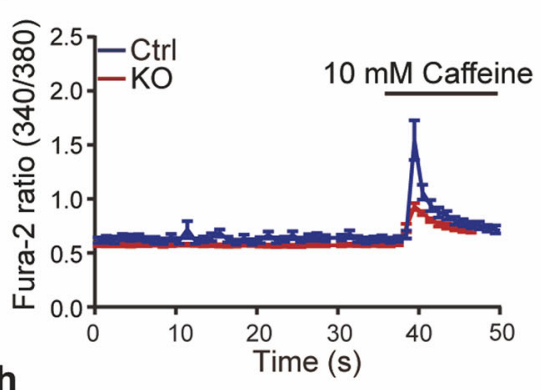

h

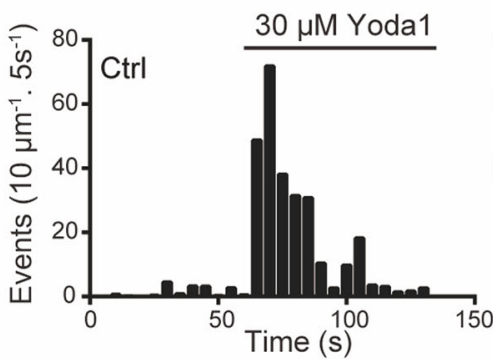

j

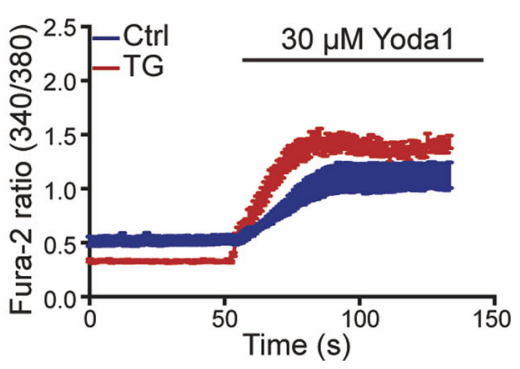

b

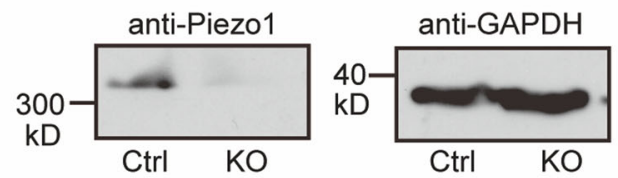

d

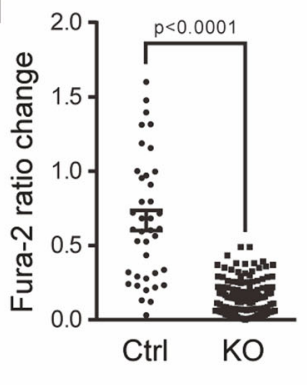

e

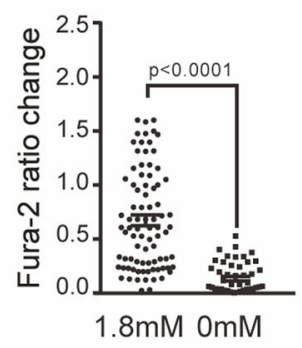

g
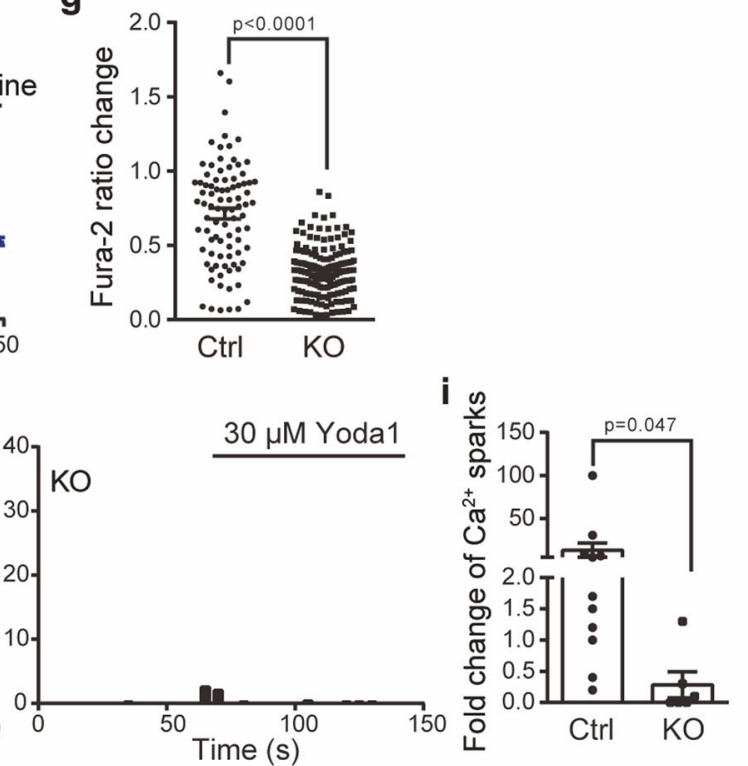

k Yoda1 response

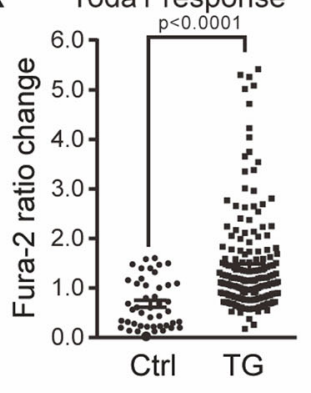

I Caffeine response

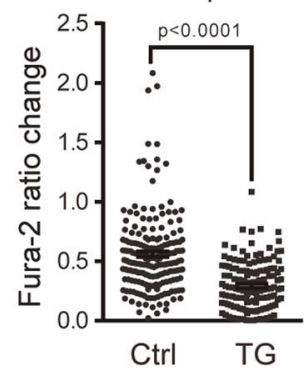

the control cells $(1.65 \pm 0.03$ vs $1.82 \pm 0.03)$ (Fig. 4b). The decrease in $\mathrm{SR} \mathrm{Ca}^{2+}$ content and spontaneous $\mathrm{Ca}^{2+}$ spark in the $\mathrm{KO}$ cells indicates that Piezo1-mediated $\mathrm{Ca}^{2+}$ influx might help to maintain the $\mathrm{SR} \mathrm{Ca}^{2+}$ store.

On the other hand, despite decreased SR $\mathrm{Ca}^{2+}$ content, the Piezo1-TG cardiomyocytes showed significantly enhanced $\mathrm{Ca}^{2+}$ spark events compared to the control cells $(3.6 \pm 0.7$ vs $1.2 \pm$ 0.2 events per $10 \mu \mathrm{m}$ per second, respectively) (Fig. $4 \mathrm{~d}$, f).
Furthermore, the peak amplitude of the $\mathrm{Ca}^{2+}$ spark from the TG cells is drastically lower than that of the control cells $(1.03 \pm 0.03$ vs $1.74 \pm 0.02$, respectively) (Fig. 4e). In addition to the frequently occurred local $\mathrm{Ca}^{2+}$ sparks, about $80 \%$ of the TG cells showed spontaneous $\mathrm{Ca}^{2+}$ waves (Fig. $4 \mathrm{~g}$, h), which represent transient but global increase in the intracellular $\mathrm{Ca}^{2+}$ that propagate as waves through the cell and are highly arrhythmogenic ${ }^{45}$. In comparison, less than $30 \%$ of the control cells showed such $\mathrm{Ca}^{2+}$ 
Fig. 2 Piezo1-dependent $\mathbf{C a}^{2+}$ response in mouse cardiomyocytes. a Scatter plot of relative Piezo1 mRNA level from the littermate control $(C t r l)(n=3)$ and cardiac-specific Piezo1-KO tissues $(n=3)$. Unpaired student's t-test, two-sided. Values are mean \pm SEM. b Representative western blotting result of Piezo1 proteins immunoprecipitated from $\mathrm{Ctrl}$ and $\mathrm{KO}$ heart homogenates using the anti-Piezo1 antibody. The GAPDH level was used for loading control. Experiment was repeated independently three times with similar results. c Representative average traces of single-cell Fura- $2 \mathrm{Ca} 2+$ imaging of $\mathrm{Ctrl}$ ( 7 cells) or KO (8 cells) cardiomyocytes in response to the Piezo1 chemical activator Yoda1. d Scatter plot of Yoda1-induced Fura-2 amplitude change of Ctrl (39 cells) and KO (127 cells) cardiomyocytes from control $(n=3)$ mice and their littermate KO $(n=4)$ mice. Unpaired student's t-test, two-sided. e Scatter plot of Yoda1-induced Fura-2 amplitude of cardiomyocytes with (84 cells) or without ( 49 cells) 1.8 mM extracellular Ca ${ }^{2+}$ from 3 mice. Unpaired student's t-test, two-sided. $\mathbf{f}$ Representative average traces of single-cell Fura-2 $\mathrm{Ca}^{2+}$ imaging of $\mathrm{Ctrl}$ ( $13 \mathrm{cells}$ ) and $\mathrm{KO}$ (20 cells) cardiomyocytes in response to 10 $\mathrm{mM}$ caffeine. $\mathbf{g}$ Scatter plot of caffeine-induced Fura-2 ratio changes from the indicated cardiomyocytes, reflecting the SR Ca ${ }^{2+}$ store level. Ctrl (89 cells) and $\mathrm{KO}\left(151\right.$ cells) from $\mathrm{KO}(n=3)$ mice and their littermate control $(n=3)$ mice. Unpaired student's t-test, two-sided. $\mathbf{h} \mathrm{Ca}^{2+}$ spark histogram before and after application of Yoda1 of Ctrl (11 cells) and KO ( 6 cells) cardiomyocytes by TIRF. Each column represents the average of $\mathrm{Ca}^{2+}$ sparks per cell in every $5 \mathrm{~s}$. i Scatter plot of fold-change of $\mathrm{Ca}^{2+}$ spark rate in Ctrl (11 cells) or KO (6 cells) cardiomyocytes from KO $(n=3)$ mice and their littermate control $(n=$ 3) mice during Yoda1-treatment. j Representative average traces of single-cell Fura-2 Ca ${ }^{2+}$ imaging of Ctrl (11 cells) or TG (20 cells) cardiomyocytes in response to Yoda1. $\mathbf{k}$ Scatter plot of Yoda1-induced Fura-2 amplitude change of Ctrl (45 cells) and TG (191 cells) cardiomyocytes from TG ( $n=4)$ mice and their littermate control $(n=3)$ mice. Unpaired student's t-test, two-sided. I Scatter plot of caffeine-induced Fura- 2 ratio changes from the indicated cardiomyocytes, reflecting the SR Ca ${ }^{2+}$ store level. Ctrl (201 cells) and TG (137 cells) from TG $(n=3)$ mice and their littermate control $(n=3)$ mice. Unpaired student's t-test, two-sided. Values are mean \pm SEM in $\mathbf{c}-\mathbf{g}, \mathbf{i}, \mathbf{k}-\mathbf{I}$.

waves (Fig. 4h). The occurring frequency of the $\mathrm{Ca}^{2+}$ waves in the TG cells is also much higher than that in the control cells $(4.6 \pm$ 1.6 vs $0.7 \pm 0.2$ per $100 \mu \mathrm{m}$ per second, respectively) (Fig. $4 \mathrm{i}$ ). Given the markedly increased resting $\mathrm{Ca}^{2+}$ sparks and waves in the TG cells, their decreased SR $\mathrm{Ca}^{2+}$ content is highly likely due to profound $\mathrm{Ca}^{2+}$ leakage from the $\mathrm{SR} \mathrm{Ca}^{2+}$ store. Collectively, these data demonstrate that Piezol has a prominent role in determining $\mathrm{Ca}^{2+}$ homeostasis in cardiomyocytes.

Piezo1 mediates stretch-induced and homeostatic ROS signaling. We next set up to understand how Piezol might affect the stretch-induced and homeostatic $\mathrm{Ca}^{2+}$ signaling in cardiomyocytes. On the one hand, Piezo1-mediated $\mathrm{Ca}^{2+}$ influx might directly contribute to cardiac $\mathrm{Ca}^{2+}$ homeostasis via the CICR mechanism. On the other hand, previous studies have suggested that stretch-induced $\mathrm{Ca}^{2+}$ sparks are mediated by a mechanochemo signaling pathway termed X-ROS signaling, which involves stretch-activation of nicotinamide adenine dinucleotide phosphate (NADPH) oxidase 2 (NOX2) via Rac1-dependent activation of microtubules, leading to the production of reactive oxygen species (ROS) that in turn modulates the activity of RyR2 ${ }^{44}$. However, whether mechanosensitive ion channels are involved in the X-ROS signaling has remained unclear. Using GsMTx4 as a blocker for mechanosensitive ion channels, one study has shown that GsMTx4 had no effect on the stretchinduced acute increase in $\mathrm{Ca}^{2+}$ spark rate ${ }^{43}$, while another study has observed blocking effect ${ }^{46}$.

We, therefore, asked whether Piezol might be involved in this $\mathrm{X}$-ROS signaling pathway by using the Piezo1-KO mouse model. To test this hypothesis, we measured ROS production in cardiomyocytes using the fluorescent ROS sensor $2^{\prime}, 7^{\prime}$-dichlorofluorescein diacetate (DCF). Interestingly, Yodal induced a significant increase in DCF fluorescence in control cardiomyocytes in the presence of extracellular $\mathrm{Ca}^{2+}$ (Fig. 5a, b). In contrast, in the absence of extracellular $\mathrm{Ca}^{2+}$, the Yodal-induced increase in DCF fluorescence was totally abolished (Fig. 5c, d). Remarkably, Piezo1-KO cardiomyocytes also totally lost the Yoda1-induced DCF response (Fig. 5e, f). Given that Yodal can induce Piezol-dependent $\mathrm{Ca}^{2+}$ influx into cardiomyocytes (Fig. 2c-e), these data suggest that Yoda1-induced increase in ROS production is mediated by Piezo1-dependent $\mathrm{Ca}^{2+}$ influx.

We next directly tested the requirement of Piezol for stretchinduced ROS production. In line with previous reports ${ }^{44}$, we observed that an $8 \%$ of stretch induced an apparent increase in DCF intensity in the control cardiomyocytes (Fig. 5g, h). Remarkably, such response was nearly completely abolished in the Piezo1-KO cardiomyocytes (Fig. 5g, h). These data provide compelling genetic evidence that Piezo1 mediates both Yoda1and stretch-induced production of ROS in cardiomyocytes. Despite that GsMTx4 is able to block heterologously expressed Piezo1, its effect on endogenously expressed Piezol in cardiomyocytes might be variable, which might account for the inconsistent results of the previous studies ${ }^{43,46}$.

Taking advantage of Yoda1-induced ROS production, we next went on to ask whether Piezol might actually serve as the direct upstream mechanotransducer of the stretch-induced X-ROS signaling pathway by providing $\mathrm{Ca}^{2+}$ influx (Fig. 5i). If this is the case, we reasoned that Yoda1-induced ROS production would be prevented by blocking the downstream Rac1 and NOX2 (Fig. 5i). Indeed, blocking the $\mathrm{Ca}^{2+}$-dependent small GTPase Rac1 with an inhibitor not only prevented Yoda1-induced ROS production as indicated by the lack of DCF fluorescence increase, but also significantly suppressed the basal level of ROS (Fig. 5j), suggesting a constitutive Racl-dependent ROS production. Furthermore, pre-incubation of the membrane-permeable NOX2 inhibiting peptide gp91ds-tat blocked Yoda1-induced production of ROS (Fig. 5k). Together, these data demonstrate that Piezo1serves as the upstream mechanotransduction channel to initiate the $\mathrm{Ca}^{2+}$ influx-Rac1-NOX2-ROS-Ca ${ }^{2+}$ spark signaling pathway (Fig. 5i).

Interestingly, in the absence of mechanical or Yoda1 stimulation, compared to the control cardiomyocytes, the Piezo1-KO cells had significantly reduced DCF fluorescence (Fig. 51), while the Piezo1-TG cells showed significantly enhanced DCF fluorescence (Fig. 5m), demonstrating that Piezol controls the ROS homeostasis in a dose-dependent manner.

Cardiac-specific deletion of Piezo1 impairs heart function. $\mathrm{Ca}^{2+}$ is essential for the cardiac excitation-contraction coupling ${ }^{11}$. Abnormal $\mathrm{Ca}^{2+}$ handling leads to heart dysfunction ${ }^{45}$. Given the critical role of Piezol in mediating cardiac $\mathrm{Ca}^{2+}$ signaling, we next examined the impact of cardiac-specific deletion of Piezol on heart function. When examined at 8-week old, the cardiac-specific Piezo1-KO mice did not display obvious defects in heart and body weights, heart morphology, and pump function (Supplementary Fig. 3a-e and Supplementary Table 1a). Furthermore, echocardiographic analysis revealed no apparent structural defects in the left ventricles of the KO mice (Supplementary Movies 1-4). Thus, the Piezo1-KO mice at 8-week old showed overall normal heart structure and function, suggesting that the MLC2v-Cre dependent deletion of Piezol might not cause developmental defects of the heart during the embryonic developmental stage. 
a
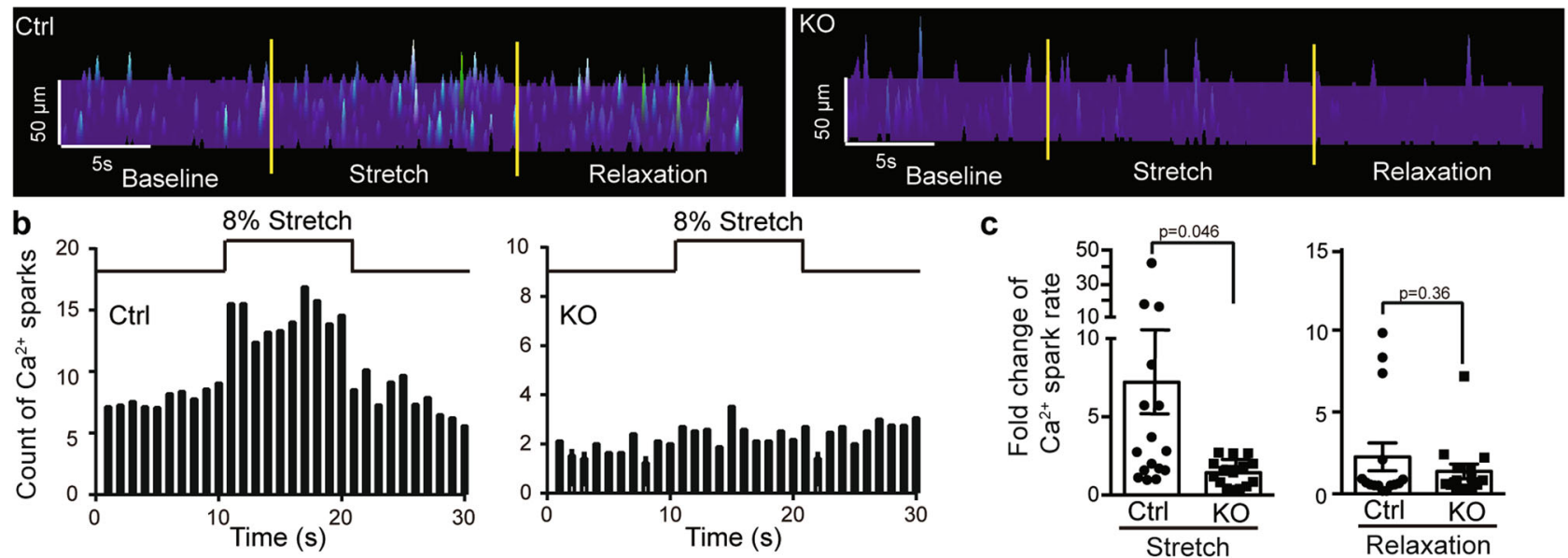

C
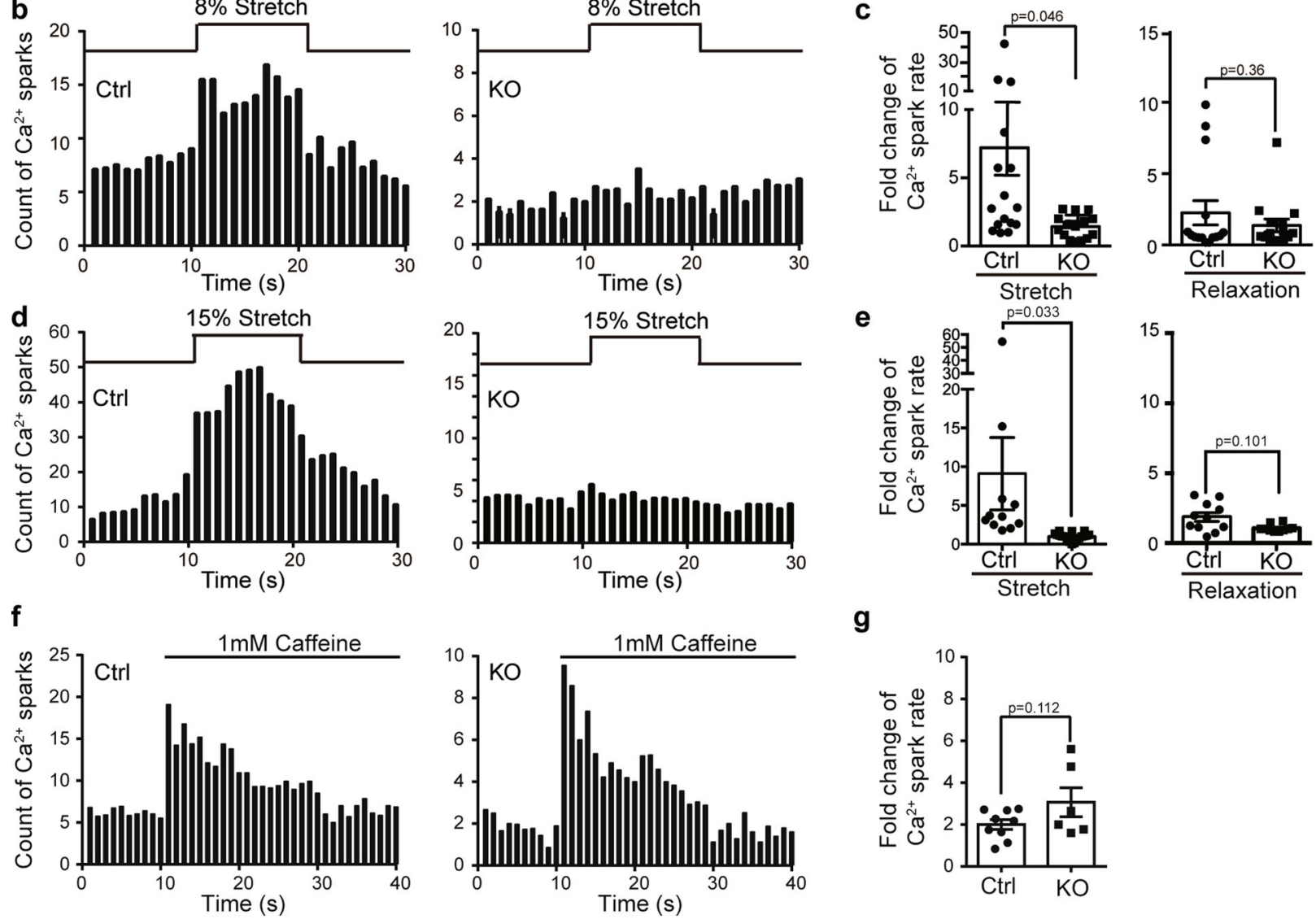

g

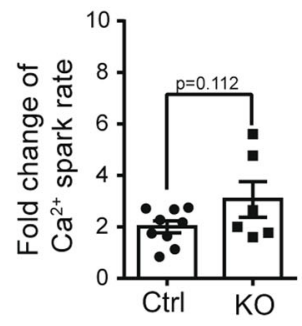

Fig. 3 Piezo1 mediates stretch-induced $\mathbf{C a}^{2+}$ signaling. a Representative fluorescence surface plot of stretch-activated $\mathrm{Ca}^{2+}$ sparks in $\mathrm{Ctrl}$ or $\mathrm{KO}$ cardiomyocytes in response to an $8 \%$ axial stretch. $\mathrm{Ca}^{2+}$ sparks were detected using line-scan mode of confocal microscopy of the $\mathrm{Ca}^{2+}$ indicator Fluo- 4 AM. b Ca ${ }^{2+}$ spark histogram before, during, and after $8 \%$ axial stretch of Ctrl (16 cells) and KO (17 cells) cardiomyocytes from KO ( $\left.n=3\right)$ mice and their littermate control $(n=3)$ mice. c Scatter plot of fold-change of $\mathrm{Ca}^{2+}$ spark rate in Ctrl (16 cells) or KO (17 cells) cardiomyocytes from KO ( $\left.n=3\right)$ mice and their littermate control $(n=3)$ mice during stretch (left panel) and upon relaxation (right panel). Unpaired student's t-test, two-sided. Values are mean \pm SEM. d Ca ${ }^{2+}$ spark histogram before, during, and after $15 \%$ axial stretch of $\mathrm{Ctrl}$ ( 11 cells) and KO (20 cells) cardiomyocytes from KO ( $n=3$ ) mice and their littermate control $(n=3)$ mice. e Scatter plot of fold-change of $\mathrm{Ca}^{2+}$ spark rate in Ctrl (11 cells) or KO (20 cells) cardiomyocytes from KO ( $\left.n=3\right)$ mice and their littermate control $(n=3)$ mice during stretch (left panel) and upon relaxation (right panel). Unpaired student's t-test, two-sided. Values are mean \pm SEM. $\mathbf{f ~ C a}{ }^{2+}$ spark histogram before and during the application of $1 \mathrm{mM}$ caffeine of Ctrl ( 9 cells) and KO (6 cells) cardiomyocytes from KO ( $n=3$ ) mice and their littermate control $(n=3)$ mice. $\mathbf{g}$ Scatter plot of fold-change of $\mathrm{Ca}^{2+}$ spark rate in Ctrl ( 9 cells) or KO ( 6 cells) cardiomyocytes from KO ( $n=3$ ) mice and their littermate control $(n=3)$ mice during application of caffeine. Unpaired student's t-test, two-sided. Values are mean \pm SEM.

At 18-week old, the $\mathrm{KO}$ mice unexpectedly had an increased body weight, but with their heart weight and the ratio of heart weight to body weight comparable to the littermate control mice (Fig. 6a-c). Histological analysis revealed that the $\mathrm{KO}$ hearts appeared moderately larger with dilated left ventricles (Fig. 6d). Masson's trichrome staining of the left ventricle sections revealed significantly enhanced intermuscular fibrosis in the KO heart sections, indicating the development of cardiomyopathy (Fig. 6e, f). Indeed, RT-PCR of the KO heart tissues verified significantly increased mRNA levels of cardiomyopathic marker genes including $\beta$-myosin heavy chain $(M H C)$ and atrial natriuretic peptide (ANP) (Fig. 6g). ECG examination did not show obvious cardiac arrhythmias in the control and KO mice (Fig. 6h). However, echocardiography showed that the KO heart had a significantly increased end-diastolic and end-systolic internal diameter and volume of left ventricle (Fig. 6i, $\mathrm{j}$ and Supplementary Table 1b). Both the diastolic and systolic volume of the left ventricle of the $\mathrm{KO}$ hearts was significantly enlarged, resulting in unchanged stroke volume (Fig. 6k and Supplementary Table $1 \mathrm{~b}$ ). The observation that a stretched diastolic left ventricle of the KO hearts $(81.8 \pm$ $8.7 \mu \mathrm{l})$ relative to that of the control hearts $(61.2 \pm 3.4 \mu \mathrm{l})$ led to a comparable stroke volume suggests a compromised stoke function and Frank-Starling response in the KO heart. The KO hearts had a concomitant decreased ejection fraction and fractional shortening (Fig. 6l, $\mathrm{m}$ and Supplementary Table 1b), demonstrating impaired heart pump function. Thus, in line with its prominent role in regulating cardiac $\mathrm{Ca}^{2+}$ handling and ROS signaling, Piezol plays a critical role in maintaining normal heart functions. 

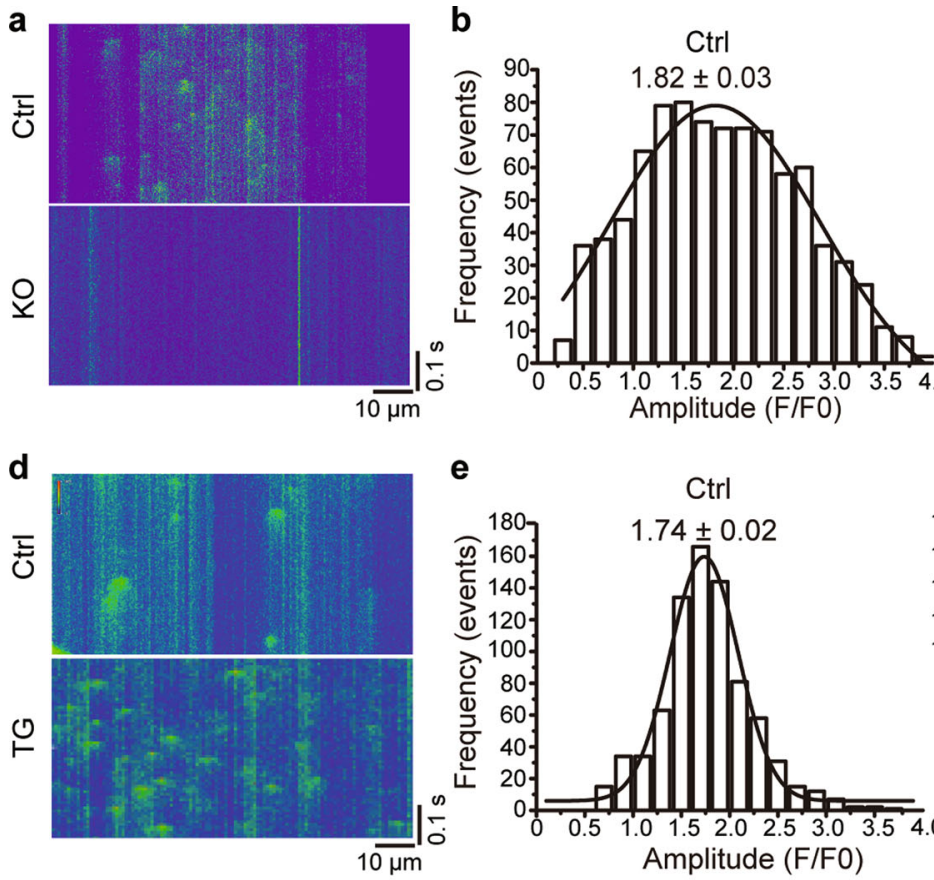

g

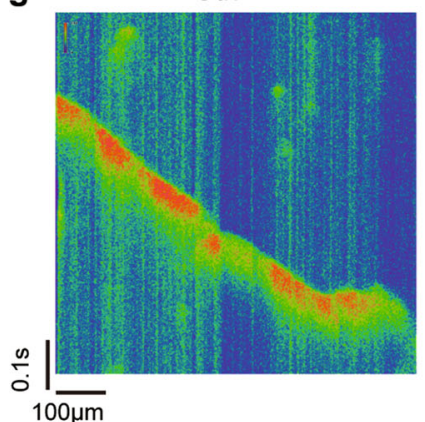

e

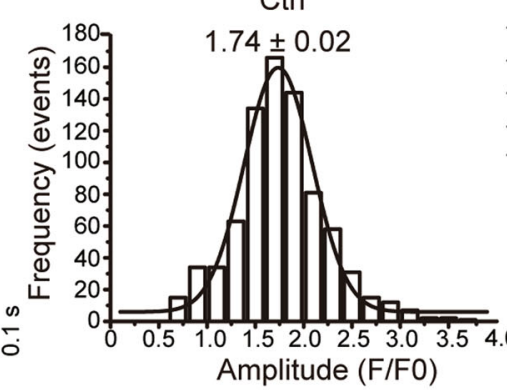

TG

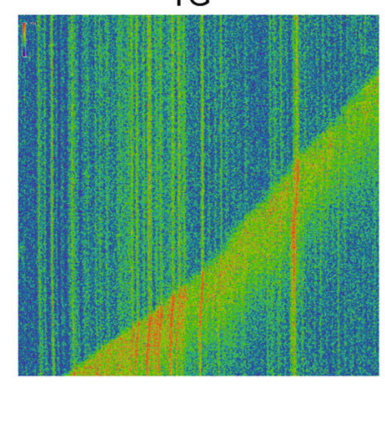

KO

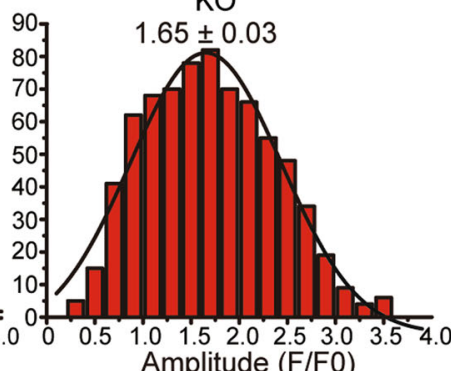

TG

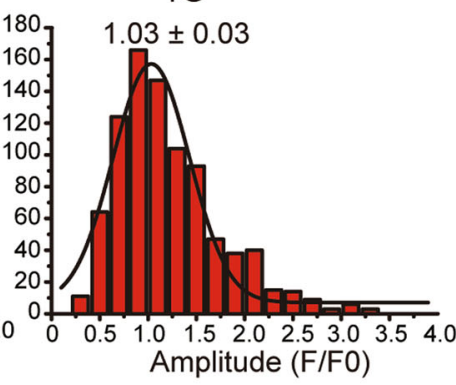

h

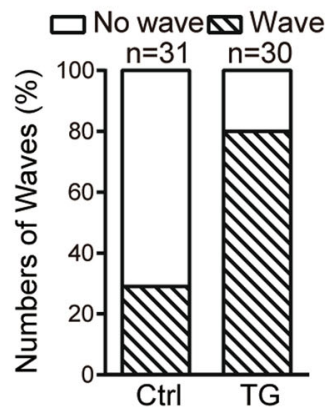

C

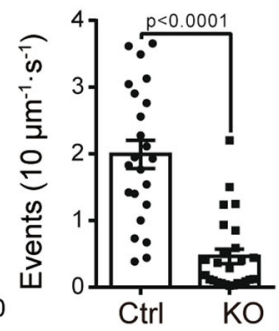

f

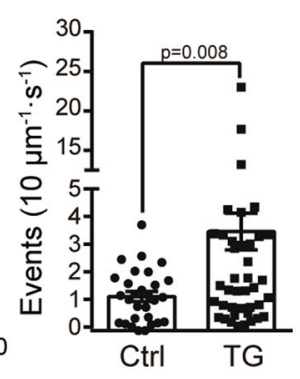

i

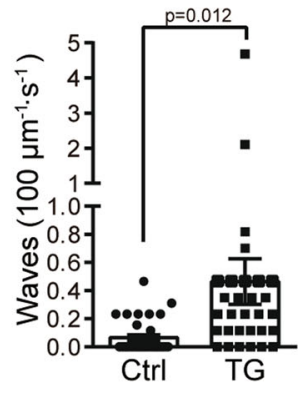

Fig. 4 Piezo1 mediates homeostatic $\mathbf{C a}^{2+}$ signaling. a, d Representative fluorescence surface plot of spontaneous $\mathrm{Ca}^{2+}$ sparks of the indicated cardiomyocytes. b, e Histogram analysis of the $\mathrm{Ca}^{2+}$ spark frequency of the indicated cardiomyocytes. The peak amplitudes are labeled above the fit. c, $\mathbf{f}$ Scatter plot of $\mathrm{Ca}^{2+}$ spark of cardiomyocytes with the indicated genotypes [ $23 \mathrm{Ctrl}$ cells and $26 \mathrm{KO}$ cells from KO $(n=5)$ mice and their littermate control $(n=5)$ mice in $\mathbf{b}, \mathbf{c} 29 \mathrm{Ctrl}$ cells and 47 TG cells from TG $(n=3)$ mice and their littermate control $(n=3)$ mice in e, f]. Unpaired student's t-test, two-sided. Values are mean \pm SEM. $\mathbf{g}$ Representative fluorescence surface plot of spontaneous $\mathrm{Ca}^{2+}$ waves of the indicated cardiomyocytes. $\mathbf{h}$ Proportion of the indicated cardiomyocytes with or without $\mathrm{Ca}^{2+}$ waves [31 Ctrl cells and 30 TG cells from TG $(n=3)$ mice and their littermate control $(n=3)$ mice]. i Scatter plot of frequency of $\mathrm{Ca}^{2+}$ waves of the indicated cardiomyocytes [31 Ctrl cells and 30 TG cells from TG $(n=3)$ mice and their littermate control $(n=3)$ mice]. Unpaired student's t-test, two-sided. Values are mean \pm SEM.

Cardiac-specific overexpression of Piezo1 induces heart failure and arrhythmias. Given the markedly increased occurrence of $\mathrm{Ca}^{2+}$ sparks and waves and decreased $\mathrm{SR} \mathrm{Ca}^{2+}$ store upon overexpression of Piezol in cardiomyocytes, we expected abnormal heart function in the cardiac-specific Piezo1-TG mice. Remarkably, compared to littermate control mice, the TG mice at 8 -week old showed significantly enlarged hearts with apparently dilated chambers without thickening of the ventricle wall (Fig. 7a-d), suggesting dilated cardiomyopathy. The morphological change of the TG heart progressed more severely with increased age. At 18-week old, the heart weight of the TG mice was nearly twice of the control heart weight $(457 \pm 46 \mathrm{mg}$ vs $223 \pm 7 \mathrm{mg}$, respectively) (Fig. 7b). The TG mice had significantly lower body weight than the control mice at both 8 -week and 18-week old (Fig. 7c). The heart weight to body weight ratio of the TG mice at 18 -week old reached about 3 folds of that of the control mice $(17.1 \pm 2.3$ vs $5.7 \pm 0.2)$ (Fig. $7 d$ ). In contrast. there was no significant difference in tibia length between the TG and control mice (Fig. 7e), indicating that the growth and size of the
TG mice was normal. The TG heart tissues also showed significantly enhanced intermuscular fibrosis (Fig. 7f, g) and increased mRNA levels of $\beta-M H C$ and $A N P$ (Fig. 7j). Remarkably, while none of the 12 littermate control mice showed arrhythmia (Fig. 7h), all the 12 TG mice examined at 4-week, 8-week or 18-week old showed ventricular tachycardia (Fig. 7h), in line with the frequently observed arrhythmogenic $\mathrm{Ca}^{2+}$ waves in the TG cardiomyocytes (Fig. 4l-n). Echocardiographic analysis of 4-week, 8-week and 18-week old mice (Supplementary Table 1c-e) showed that the TG mice had severely impaired heart pump function as reflected by their significantly increased internal diameter and volume of left ventricle (Fig. 7k-n) and a markedly decreased ejection fraction and fractional shortening (Fig. 7p, q). However, echocardiographic analysis revealed no structural defects in the left ventricle of TG mice (Supplementary Movies 5-8), indicating that the defective pump function of the TG mice might not be due to developmental defects. Together, these characterizations demonstrate that cardiac-specific overexpression of Piezol leads to severe heart failure and arrhythmias, 

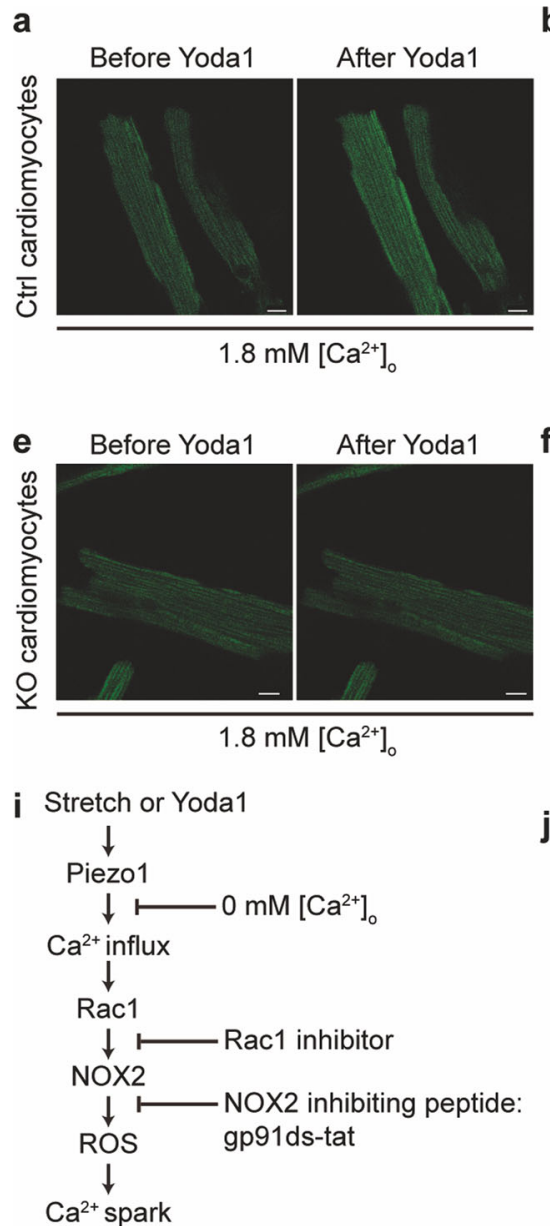
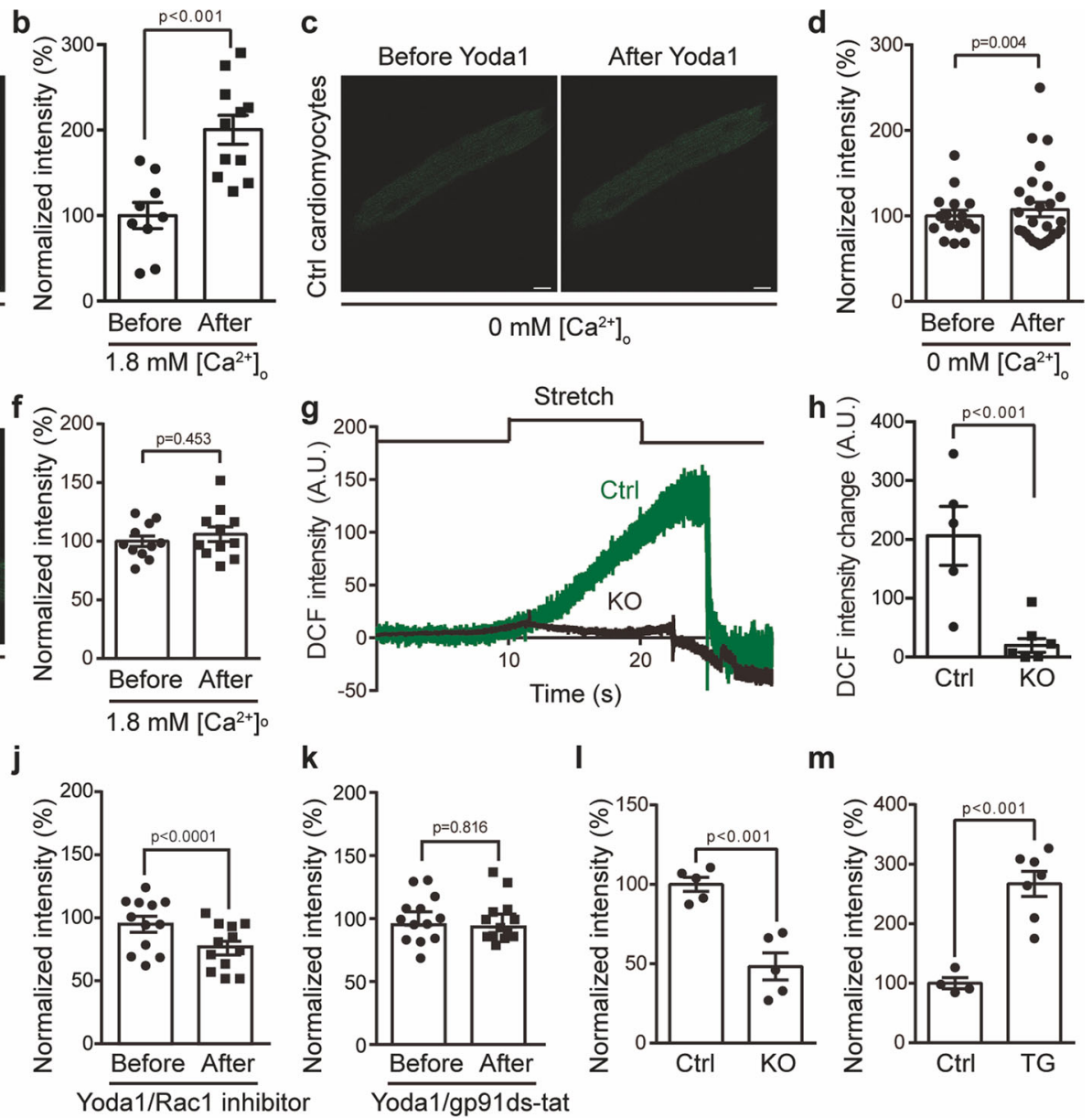

m
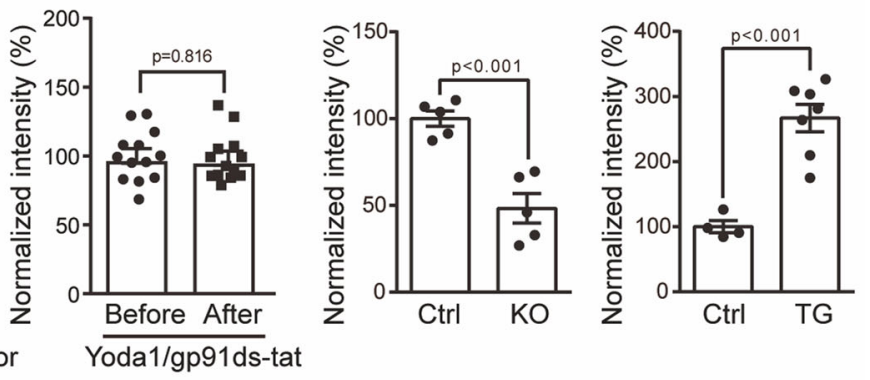

Fig. 5 Piezo1 mediates stretch-induced and homeostatic ROS signaling. a, c Representative DCF fluorescent images of Ctrl cardiomyocytes before and after application of Yoda1 in the presence (a) or absence (c) of $1.8 \mathrm{mM}\left[\mathrm{Ca}^{2+}\right]_{0}$. Scale bar, $10 \mu \mathrm{m} . \mathbf{b}$, d Scatter plot of normalized DCF intensity of Ctrl cardiomyocytes before and after application of Yoda1 in the presence (11 cells) or absence ( 15 cells) of $1.8 \mathrm{mM}\left[\mathrm{Ca}^{2+}\right]_{0} .3 \mathrm{mice}$ for each group. Paired student's t-test, two-sided. e Representative DCF fluorescent images of $\mathrm{KO}$ cardiomyocytes before and after application of Yoda1 in the presence of $1.8 \mathrm{mM}$ $\left[\mathrm{Ca}^{2+}\right]_{0}$. Scale bar, $10 \mu \mathrm{m}$. $\mathbf{f}$ Scatter plot of normalized DCF intensity of $\mathrm{KO}$ cardiomyocytes (11 cells from $3 \mathrm{KO}$ mice) before and after application of Yoda1 in the presence of $1.8 \mathrm{mM}\left[\mathrm{Ca}^{2+}\right]_{0}$. Paired student's t-test, two-sided. $\mathbf{g}$ Representative DCF fluorescent imaging trace of Ctrl or KO cardiomyocytes in response to $8 \%$ axial stretch. $\mathbf{h}$ Scatter plot of stretch-induced DCF fluorescence intensity change of Ctrl ( 5 cells) or KO cardiomyocytes (6 cells). 3 mice for each group. Unpaired student's t-test, two-sided. $\mathbf{i}$ The proposed scheme of Piezo1-mediated ROS-generating pathway. $\mathbf{j}$ Scatter plot of normalized DCF intensity of wild-type cardiomyocytes (12 cells from 3 mice of three independent experiments) in response to Yoda1 together with the Rac1 inhibitor. Paired student's t-test, two-sided. $\mathbf{k}$ Scatter plot of normalized DCF intensity of wild-type cardiomyocytes (14 cells from 3 mice of three independent experiment) in response to Yoda1 after pre-incubation with the membrane-permeable NOX2 inhibiting peptide gp91ds-tat. Paired student's t-test, two-sided. I Scatter plot of normalized DCF intensity of Ctrl ( 5 cells) and KO ( 5 cells) cardiomyocytes for three independent experiments. Unpaired student's t-test, two-sided. m Scatter plot of normalized DCF intensity of Ctrl (4 cells) and TG (7 cells) cardiomyocytes from three independent experiments. Unpaired student's t-test, two-sided. Values are mean \pm SEM in $\mathbf{b}, \mathbf{d}, \mathbf{f}, \mathbf{h}, \mathbf{j}-\mathbf{m}$.

consistent with abnormal $\mathrm{Ca}^{2+}$ handling and ROS signaling in the Piezo1-TG cardiomyocytes.

Autonomic upregulation of Piezol contributes to the development of cardiomyopathy. Given that both deletion and overexpression of Piezol can lead to heart dysfunction in mice (Figs. 6, 7), we wondered the pathological contribution of Piezol to cardiomyopathy. A previous study has suggested upregulation of Piezol in myocardial infarction-induced failing rat hearts ${ }^{34}$. Our studies of the mechanotransduction of osteoblasts have revealed a positive feedback loop between mechanical force and the Piezol mechanosensor itself, in which a mechanical load leads to increased expression of Piezo1 ${ }^{47}$. To examine whether heart might possess a similar positive feedback mechanism for regulating Piezol expression in response to mechanical stress such as during the development of cardiomyopathy, we subjected the Piezo1-Flag-KI mice for doxorubicin-induced dilated cardiomyopathy ${ }^{48}$. Western blotting of the anti-Flag-immunoprecipitated samples from cardiomyocytes derived from doxorubicin-treated Piezo1-Flag-KI mice revealed more abundant Piezol proteins than that from the saline-treated Piezo1-Flag-KI mice (Fig. 8a). The sample from saline-treated wildtype control mice was used as a negative control for demonstrating the Piezo1-specific western blotting signal (Fig. 8a). Consistent with upregulation of Piezol proteins in diseased mouse and rat hearts (Fig. 8a $)^{34}$, RT-PCR revealed a 5 -fold increase in the mRNA expression of Piezol in human heart samples with hypertrophic cardiomyopathy compared to normal heart samples (Fig. $8 \mathrm{~b}$ and Supplementary Excel Sheet). Thus, Piezol is upregulated under pathological conditions.

To test whether the upregulation of Piezol expression in diseased hearts might be due to an autonomic response of cardiomyocytes, we subjected cardiomyocytes derived from the 

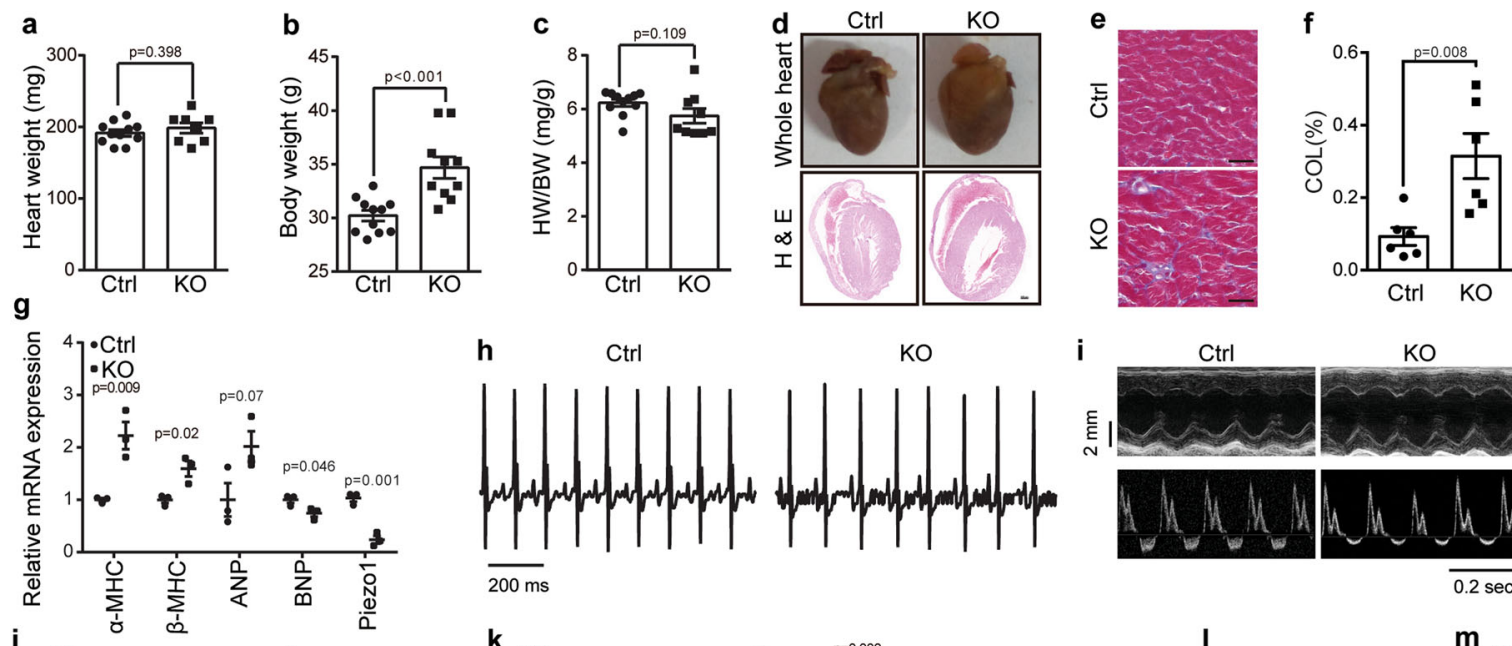

h $\quad$ Ctrl

KO

i

Ctrl
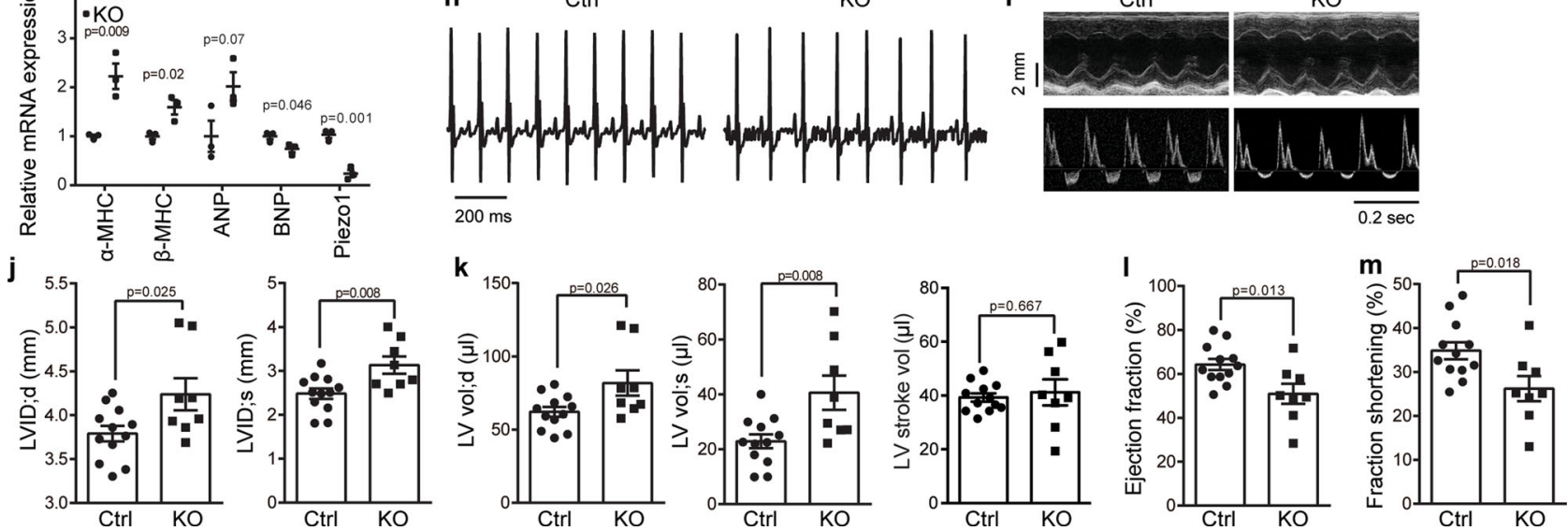

Fig. 6 Cardiac specific knockout of Piezo1 impairs heart function. a-c Scatter plot of heart weight (a), body weight (b), and HW (heart weight)/BW (body weight) ratio (c) of 18-week old littermate $\mathrm{Ctrl}(n=11)$ and Piezo1-KO $(n=10)$ male mice. Unpaired student's t-test, two-sided. Values are mean \pm SEM. d Histologic analysis of whole hearts or $\mathrm{H} \& \mathrm{E}$ stained longitudinal heart sections derived from 18-week old Ctrl and KO male mice. e Histologic analysis of the left ventricles of the Ctrl and KO hearts sectioned longitudinally and subjected to Masson's trichrome staining. Scale bar, $20 \mu \mathrm{m}$. $\mathbf{f}$ Scatter plot of collagen content in the ventricular area of the Ctrl and KO hearts derived from 18-week old mice $(n=6)$. Unpaired student's t-test, two-sided. Values are mean \pm SEM. $\mathbf{g}$ RT-qPCR analysis of the normalized mRNA level of the indicated cardiac genes derived from 18 -week old Ctrl and KO mice $(n=3)$. Unpaired student's t-test, two-sided. Values are mean \pm SEM. h Representative ECG recordings from 18-week old Ctrl and KO male mice. i Top panel: M-mode parasternal short-axis at mid-ventricular level of end-diastolic and end-systolic dimension of left ventricle; Lower panel: Pulsed Doppler at mitral valve inflow showing mitral blood flow velocity of early-diastolic and end-diastolic. j-m Scatter plots of echocardiographic analysis of left ventricular enddiastolic internal diameter (LVID;d) (left panel) and end-systolic internal diameter (LVID;s) (right panel) (j); diastolic (left panel) systolic (middle panel), and stroke volume (right panel) of left ventricle (k); percentage of ejection fraction (I); percentage of fractional shortening ( $\mathbf{m}$ ) determined from transthoracic M-mode tracings from 18-week old Ctrl $(n=12)$ and KO $(n=8)$ mice. Unpaired student's t-test, two-sided. Values are mean \pm SEM. All experiments were conducted with male mice.

littermate control and the Piezo1-KO neonatal hearts to the hypertrophic agonist phenylephrine (PE) for modeling cellular hypertrophy ${ }^{49}$. PE-treated control myocytes showed increased expression of ANP (Fig. 8c), verifying the hypertrophic effect of PE. Consistent with the observed upregulation of Piezol in diseased heart tissues (Fig. 8a, b), the mRNA expression of Piezo1 was also significantly increased in PE-treated control myocytes compared to non-treated control cells (Fig. 8c), suggesting an autonomic upregulation of Piezol in cardiomyocytes. Intriguingly, while the $\mathrm{KO}$ myocytes without PE treatment showed higher expression of ANP and $\beta$-MHC than control myocytes without PE treatment, PE-treated KO cells unexpectedly had reduced expression of ANP and $\beta$-MHC (Fig. 8c). These data indicate that deletion of Piezol appears to reverse PE-induced hypertrophy of cardiomyocytes. Together with the observation that cardiac-specific Piezol-TG mice displayed severe heart failure (Fig. 7), these data collectively demonstrate that maladaptive upregulation of Piezol in response to cardiac perturbation might contribute to the pathogenesis of cardiomyopathy in both mouse and human hearts.

\section{Discussion}

Piezol is a bona fide mechanosensitive cation channel that utilizes its unique three-bladed, propeller-like architecture to effectively convert distinct forms of mechanical stimuli into $\mathrm{Ca}^{2+}$ signaling in various cell types ${ }^{14,50}$, and consequently plays critical roles in various aspects of vascular physiology, including blood and lymphatic vessel development, vascular tone, arterial remodeling, and baroreflex control of blood pressure and heart rate $^{16,18,27}$. Cardiomyocytes experience drastic mechanical changes on a beat-to-beat basis ${ }^{1}$, and their mechanical responses are believed to underlie the well-known Frank-Starling law and Anrep effect that constitute powerful mechanisms to allow the heart to adapt to an abrupt rise in either preload or afterload ${ }^{4}$. Stretch-induced increase in intracellular $\mathrm{Ca}^{2+}$ underlies a fundamental mechanism for stretch-dependent change in the development of mechanical force of cardiomyocytes. Here we have established that the mechanosensitive Piezol channel serves as a key cardiac mechanotransducer that directly converts mechanical stretch of cardiomyocytes into $\mathrm{Ca}^{2+}$ and ROS signaling (Figs. 1-5), providing a crucial molecular basis underlying the positive relationship between mechanical stress and cardiac force production.

The observation that Piezol controls both stretch-induced and homeostatic $\mathrm{Ca}^{2+}$ and ROS signaling might suggest a Piezo1mediated positive feedback loop between these two signaling molecules critical for cardiac function. Piezol-dependent $\mathrm{Ca}^{2+}$ influx regulates the production and homeostasis of ROS via the Rac1-NOX2 signaling pathway, which might, in turn, sensitize $\mathrm{Ca}^{2+}$ release via acting on RyR2, whose sensitivity can be modulated through ROS-mediated posttranslational modification $s^{51,52}$. Such a positive feedback signaling transduction mechanism might 
a

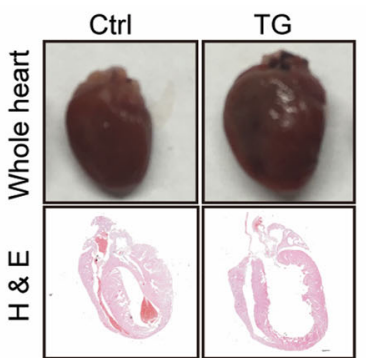

e

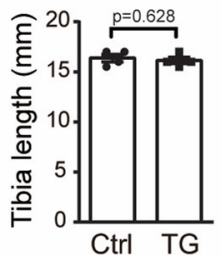

b

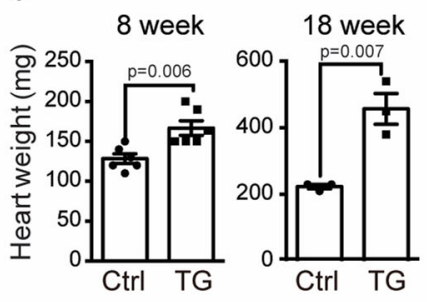

C

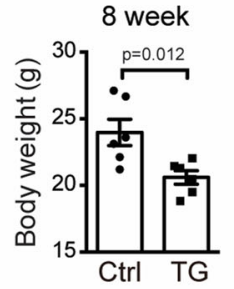

d

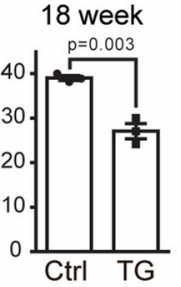

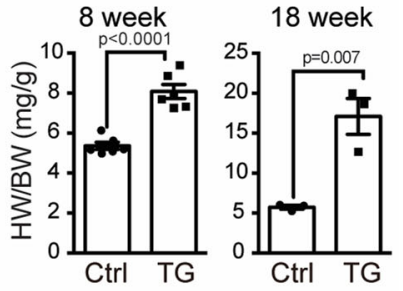

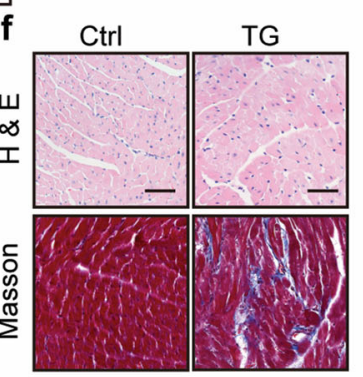

g

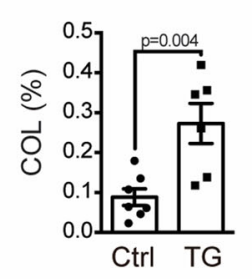

h

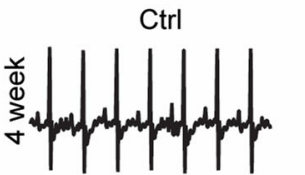

TG
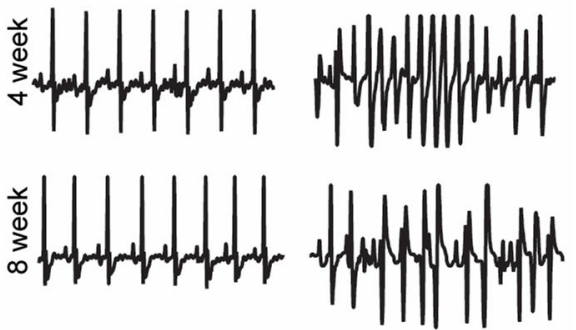

i

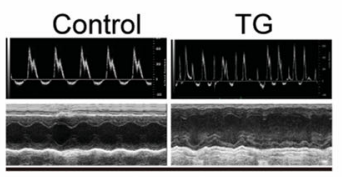

4 week

Control

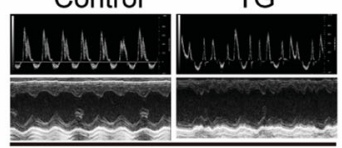

8 week

$\mathbf{k}$
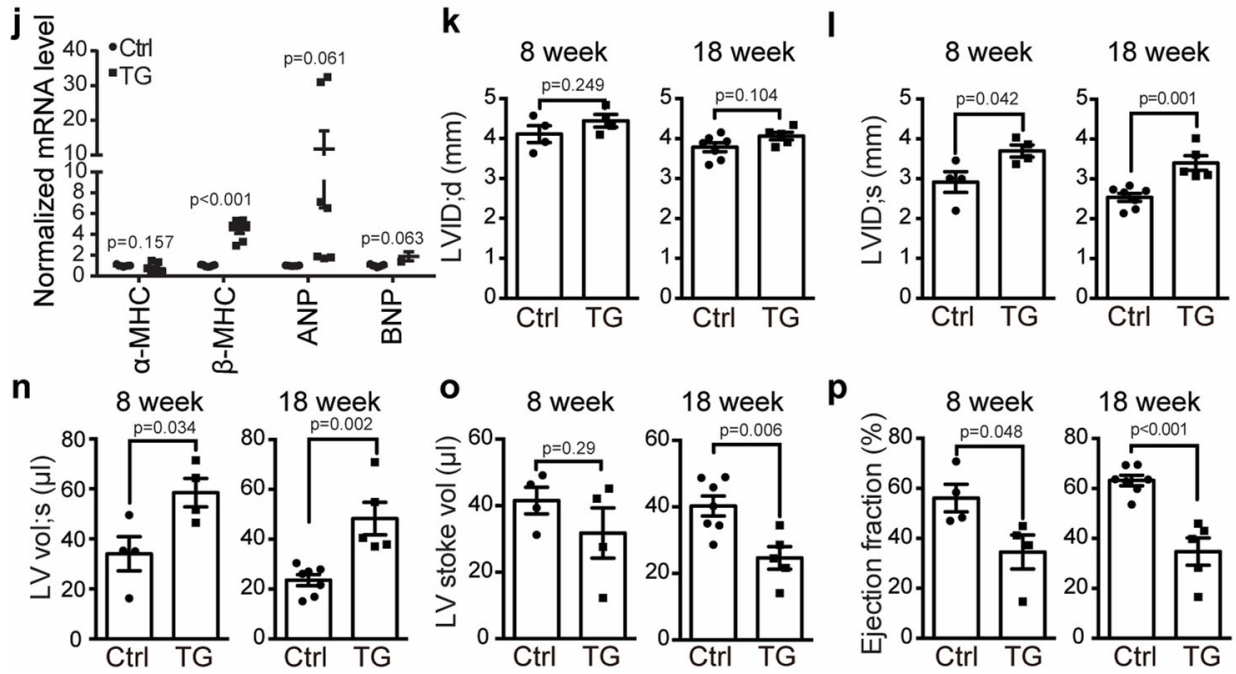

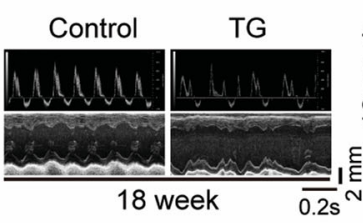

I
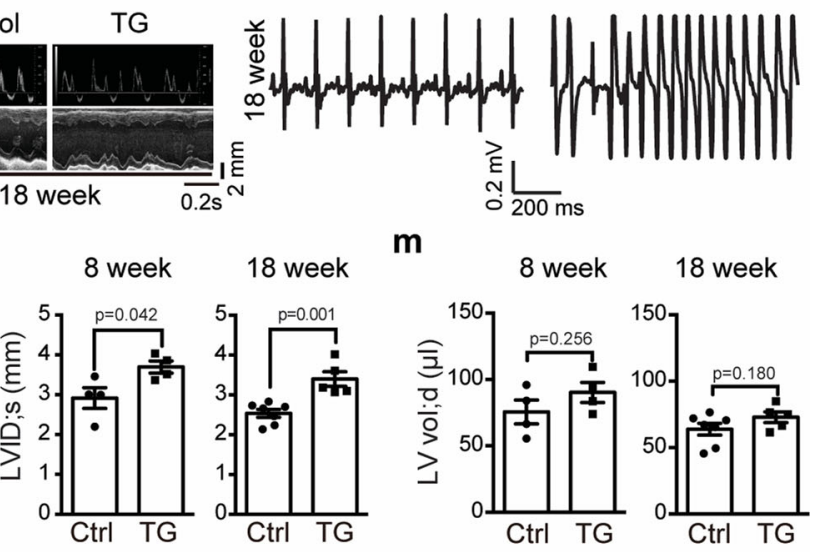

m

Fig. 7 Cardiac-specific overexpression of Piezo1 induces severe heart failure and arrhythmias. a Histologic analysis of whole hearts or $\mathrm{H} \& \mathrm{E}$ stained longitudinal heart sections derived from 8-week old littermate control (Ctrl) and Piezo1-TG male mice (TG). b-d Scatter plots of heart weight (b), body weight (c), and HW/BW ratio (d) of 8-week-old ( $n=6$ mice for each group) or 18-week old ( $n=3$ mice for each group) Ctrl and TG male mice. Unpaired student's t-test, two-sided. Values are mean \pm SEM. e Scatter plots of tibia length of 8 -week old ( $n=4$ mice for each group) Ctrl littermates and TG male mice. Unpaired student's t-test, two-sided. Values are mean \pm SEM. $\mathbf{f}$ Histologic analysis of the left ventricles of 8-week old Ctrl and TG hearts sectioned longitudinally and subjected to either H \& E (top panel) or Masson's trichrome staining (lower panel). Scale bar, $20 \mu \mathrm{m}$. $\mathbf{g}$ Scatter plot of collagen content in the ventricular area of 8-week old Ctrl and TG hearts ( $n=6$ for each group). Unpaired student's t-test, two-sided. Values are mean \pm SEM. h Representative ECG recordings from 4-week, 8-week, or 18-week old Ctrl and TG male mice. i Representative echocardiographs of 4-week, 8-week, and 18-week old Ctrl and TG littermates. $\mathbf{j}$ RT-PCR analysis of the normalized mRNA level of the indicated cardiac genes derived from the Ctrl and TG mice $(n=7)$. Unpaired student's t-test, two-sided. Values are mean \pm SEM. k-q Scatter plots of echocardiographic analysis of the indicated parameters from 8-week ( $n=4$ mice for each group) or 18-week ( $n=4$ mice for each group) Ctrl and TG mice. Unpaired student's t-test, two-sided. Values are mean \pm SEM. All experiments were conducted with male mice.

allow a relatively low abundant but well sarcolemma-distributed Piezol to optimally respond to the drastic and repeated mechanical stress generated by the beating heart. Indeed, either deletion or overexpression of Piezol resulted in dysregulated $\mathrm{Ca}^{2+}$ and ROS signaling and heart dysfunction (Figs. 6, 7). Piezol-mediated $\mathrm{Ca}^{2+}$ influx might also contribute to maintain the $\mathrm{SR} \mathrm{Ca}^{2+}$ store. Thus, we propose that the Piezol-mediated mechano-chemo transduction process might safeguard a homeostatic functional state of the heart. Disrupting such a homeostatic role might cause abnormal contractile function and arrhythmias. For instance, breaking the positive feedback loop upon Piezol deletion leads to a decreased $\mathrm{Ca}^{2+}$ influx and ROS production, resulting in a combination of reduced $\mathrm{SR} \mathrm{Ca}^{2+}$ content (Fig. 2f, g) and unsensitized SR $\mathrm{Ca}^{2+}$ release (Fig. $4 a-c)$, which is expected to cause comprised heart pump 
a

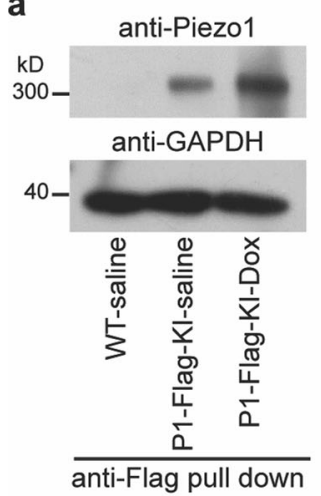

b

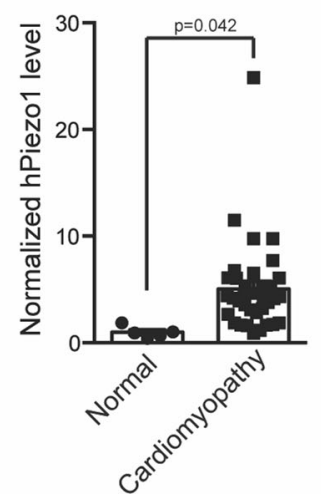

C

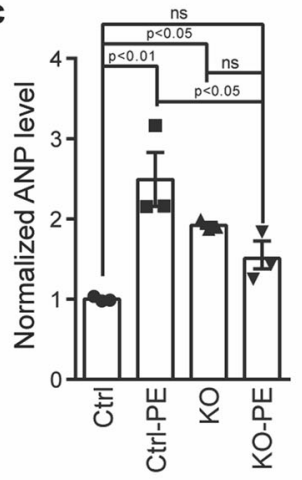

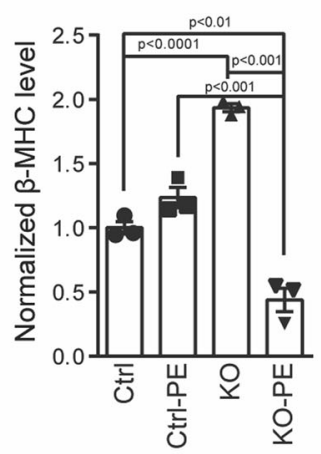

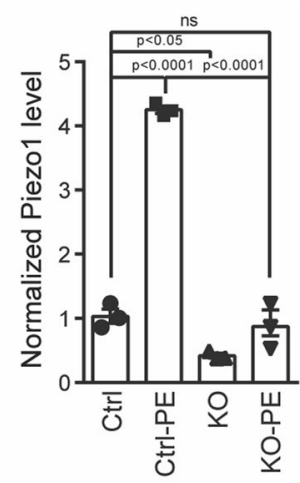

Fig. 8 Autonomic upregulation of Piezo1 contributes to the development of cardiomyopathy. a Western blotting of the anti-Flag-pulled-down Piezo1 proteins from adult cardiomyocytes derived from saline-treated wild-type mice or the Piezo1-Flag-KI mice treated with or without doxorubicin (Dox), which was used for inducing dilated cardiomyopathy. Similar results were obtained from 4 independent experiments. $\mathbf{b}$ RT-PCR analysis of the normalized mRNA level of human Piezo1 derived from either normal human hearts $(n=5)$ or hearts with hypertrophic obstructive cardiomyopathy ( $n=35)$. Unpaired student's t-test. Values are mean \pm SEM. c RT-PCR analysis of the normalized mRNA level of the cardiac hypertrophic genes and Piezo1 in primary neonatal ventricular myocytes derived from either control or Piezo1-KO mice treated with or without phenylephrine (PE) for $24 \mathrm{~h}$, which was used for inducing cardiac hypertrophy. mRNA levels were normalized by GAPDH levels. Data from 3 independent experiments. One-way ANOVA. Values are mean \pm SEM.

function and the development of cardiomyopathy of the Piezo1-KO mice (Fig. 6). On the other hand, enforcing the positive feedback loop upon overexpression of Piezol might initially cause increased $\mathrm{Ca}^{2+}$ influx (Fig. 2j, k) and ROS production (Fig. $5 \mathrm{~m}$ ), resulting in enhanced spontaneous SR $\mathrm{Ca}^{2+}$ leakage (Fig. 4d, f), deceased SR $\mathrm{Ca}^{2+}$ store (Fig. $2 \mathrm{i}$ ) and occurrence of arrhythmogenic $\mathrm{Ca}^{2+}$ waves (Fig. 4g-i). Such a molecular mechanism might account for the severe heart failure and arrhythmia of the Piezol-TG mice (Fig. 7).

A positive feedback relationship between the Piezol mechanosensor and the mechanical force experienced by the mechanosensitive cells and organs has been illustrated in several biological settings, in which a mechanical load leads to increased expression and activation of Piezo1. For instance, in glioma tissues, activation of Piezol by mechanical force upregulated extracellular matrix and stiffened the tissue, which in turn elevated Piezol expression to promote glioma aggression ${ }^{53}$. In osteoblasts, mechanical loading (e.g., mice were subjected to exercise on a treadmill) led to increased expression and activation of Piezol, which in turn enhanced osteogenesis and bone formation. In reverse, either mechanical unloading (e.g. hind-limb suspension or microgravity treatment) or loss of the Piezol mechanosensor (e.g. osteoblastspecific knockout) resulted in impaired osteogenesis and bone loss ${ }^{47}$. A similar positive feedback mechanism might also exist between Piezol and the mechanical force experienced by cardiomyocytes. Indeed, an autonomic upregulation of Piezol in cardiomyocytes contributes to cardiomyopathy in both cellular and animal models, as well as in human patients, which might have altered mechanical stress conditions (Fig. 8a-c). Presumably, an upregulation of Piezo1 might initially serve as an adaptive response to meet the mechanical load of the heart under pathological or aging conditions, but eventually lead to cardiomyopathy due to the positive feedback mechanism on $\mathrm{Ca}^{2+}$ and ROS signaling. Despite that the exact reason for the age-dependent phenotype remains unclear, we currently speculate that such a hypothesis might explain why only Piezo1-KO mice at 18-week old but not at 8-week old developed cardiomyopathy (Fig. 6). On the other hand, deletion of Piezol reversed PE-induced deterioration of cardiomyoctes (Fig. 8c). Thus, blocking Piezo1 by disrupting the positive feedback loop during pathogenesis might represent a therapeutic strategy for treating human heart diseases.

Previous studies have indicated the contribution of other $\mathrm{Ca}^{2+}$ signaling pathways to the development of the slow force response
(SFR) of the Anrep effect ${ }^{4}$. For instance, on the basis of that pharmacological blocking of the $\mathrm{Na}^{+} / \mathrm{H}^{+}$exchange (NHE1) reduced the SFR by $\sim 50 \%$, it has been hypothesized that stretchdependent modulation of NHE1 increases intracellular $\mathrm{Na}^{+}$, which in turn activates the reverse mode of the $\mathrm{Na}^{+} / \mathrm{Ca}^{2+}$ exchange (NCX) to increase intracellular $\mathrm{Ca}^{2+}$, leading to the development of $\mathrm{SFR}^{54}$. However, it remains unclear whether NHE1 directly respond to mechanical stimulation or indirectly affects the bona fide cardiac mechanotransducer. Piezol has been shown to be inhibited by low $\mathrm{pH}^{55}$. One conceivable scenario is that inhibition of NHE1 results in decreased $\mathrm{pH}$, which in turn inhibits stretchinduced and Piezol-mediated $\mathrm{Ca}^{2+}$ influx. Furthermore, as a cationic channel, Piezol conducts $\mathrm{Na}^{+}$as well, raising the possibility that Piezol might help to raise intracellular $\mathrm{Na}^{+}$and consequently activate the reverse mode of NCX to increase intracellular $\mathrm{Ca}^{2+}$. Alternatively, Piezo1 and NHE1-NCX might function in parallel mechanotransduction pathways. Indeed, inhibition of NHE1 and NCX only suppressed $\sim 50 \%$ of the SFR. Future studies might dissect out the relationship between Piezol and other molecules involved in cardiac mechanotransduction.

\section{Methods}

Mice. The laboratory animal facility has been accredited by Association for Assessment and Accreditation of Laboratory Animal Care International (AAA LAC) and the Institutional Animal Care and Use Committee (IACUC) of Tsinghua University approved all animal protocols used in this study.

To generate the Piezo1-Flag knock-in reporter mice (Piezo1-Flag-KI), we knocked in the Flag tag after the coding sequence corresponding to G2420 of mouse Piezo1 using the CRISPR/Cas9 technology. T7-Cas9 PCR product and T7sgRNA were gel purified and used as the template for in vitro transcription. Cas 9 mRNA, sgRNA, and oligo were mixed and injected into the cytoplasm of one-cell fertilized eggs ${ }^{56}$. Two pairs of primers were designed for genotyping the genomic DNA derived from mouse tails of the Piezol-Flag-KI breeding mice. One pair was used to distinguish between the wild-type and Flag-inserted bands, while the other pair was to verify the insertion of the Flag-tag sequence into the genome (Supplementary Table 2).

The previously described floxed Piezol mice (Piezo1 ${ }^{\mathrm{fl} / \mathrm{fl}}$ ) ${ }^{24}$ (a generous gift from Dr. Ardem Patapoutian at the Scripps Research) were crossed with the MLC2v-Cre mice ${ }^{39}$, in which the Cre recombinase coding sequence was knocked into the

MLC2v genomic locus that is expressed exclusively in ventricular cardiomyocytes to obtain Piezol $1^{\mathrm{fl} / \mathrm{fl}}$ as littermate control (Ctrl) and Piezo1 ${ }^{\mathrm{fl} / \mathrm{fl} / \mathrm{MLC} 2 \mathrm{v}-\mathrm{Cre} \text { as }}$ cardiac-specific Piezol-knockout (KO) mice for experimental tests (Supplementary Fig. 2a). The previously generated Piezol-TGl-mCherry-stop-fl mice ${ }^{40}$ were mated with the MLC2v-Cre mice to obtain the cardiac-specific Piezol transgenic mice

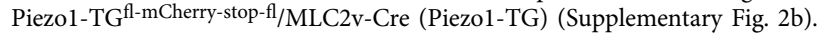


Doxorubicin-induced mouse dilated cardiomyopathy (DCM) model ${ }^{48}$ was made with intraperitoneal (i.p.) injection of doxorubicin hydrochloride (Worthington) at a dose of $3 \mathrm{mg} / \mathrm{kg}$ on 8 -week-old male mice for six times over 2 weeks. One month after the last i.p. injection, mice that had a left ventricular internal diastolic diameter (LVIDd) larger than $3.8 \mathrm{~mm}$ or $120 \%$ of that before the injection were used as the DCM models in these experiments.

All experiments were conducted using male mice in C57/BL6 genetic background.

Primary culture of mouse cardiomyocytes. Adult ventricular cardiomyocytes were isolated from wild-type C57/BL6, Piezo1-Flag-KI, Piezo1-tdTomato-KI, Piezo1-KO, Piezo1-TG, and their littermate control mice. Mice were anesthetized by injection of tribromoethanol. The heart was quickly separated and perfused in a Langendorff apparatus with a zero $\mathrm{Ca}^{2+}$ Tyrode's solution for $3 \mathrm{~min}$ at $37^{\circ} \mathrm{C}$, then digested with $50 \mu \mathrm{M} \mathrm{Ca}^{2+}$ Tyrode's solution containing $0.9 \mathrm{mg} / \mathrm{mL}$ type II collagenase (Worthington, USA) and $0.05 \mathrm{mg} / \mathrm{ml}$ protease for about $20 \mathrm{~min}$. All buffers contained $10 \mathrm{mM}$ 2,3-butanedione monoxime (BDM) for suppressing contraction. Adult mouse cardiomyocytes were mechanically separated and gradually recovered into $1.8 \mathrm{mM} \mathrm{Ca}^{2+}$ Tyrode's solution and plated on glass coverslips coated with laminin.

Neonatal mouse cardiomyocytes were isolated from neonatal mice 1-3 days after birth. After decapitation, the heart was extracted and its ventricular section was cut into pieces and sequentially digested with $0.125 \%$ Trypsin at $4{ }^{\circ} \mathrm{C}$ for $30 \mathrm{~min}$ and then with $0.5 \%$ collagenase II at $37^{\circ} \mathrm{C}$ for $30 \mathrm{~min}$. All buffers contained $20 \mathrm{mM}$ BDM. Tissue fragments were filtered by a cell-strainer. The resulting cells were pre-plated for $1-2 \mathrm{~h}$ to allow fibroblasts and endothelial cells to attach to the culture dish. Non-adherent cardiomyocytes in the suspension were collected and transferred into a laminin-coated cell culture dish ${ }^{57} .24 \mathrm{~h}$ later, $100 \mu \mathrm{M}$ of phenylephrine (PE) or vehicle was added to the cell culture, which was then maintained for an additional $24 \mathrm{~h}$ to induce cellular hypertrophy.

Quantitative Real time PCR (RT-qPCR). Total RNA isolated from whole hearts, lungs, blood vessels, or cultured adult mouse cardiomyocytes was subjected to reverse transcription using the reverse transcription kit (Takara). Reactions were run and analyzed using a CFX96 Touch PCR instrument (Bio-Rad) with GAPDH as an endogenous internal control. All primers used were shown in Supplementary Table 2. Relative expression values for each gene were calculated using the $\Delta \Delta \mathrm{Ct}$ analysis method ${ }^{58}$

Immunoprecipitation and western blotting. Cell lysates derived from isolated adult cardiomyocytes were prepared using a buffer containing $25 \mathrm{mM}$ NaPIPES, $140 \mathrm{mM} \mathrm{NaCl}, 1 \%$ CHAPS, $0.5 \%$ phosphatidylcholine (PC), and a cocktail of protease inhibitors (Roche). For immunoprecipitation using the anti-Flag antibody, cell lysates were incubated with magnetic beads conjugated with the anti-Flag antibody at $4{ }^{\circ} \mathrm{C}$ for overnight. For immunoprecipitation using the anti-Piezol antibody that was custom generated by Abgent (Suzhou, China) and previously characterized ${ }^{35}$, the protein A/G magnetic beads (Cell Signaling Technology) were incubated with either IgG or the anti-Piezol antibody at $4^{\circ} \mathrm{C}$ for $2 \mathrm{~h}$. Then the antibody-bound beads were incubated with the cell lysates at $4{ }^{\circ} \mathrm{C}$ for overnight. The immunoprecipitated beads were washed 5 times with a wash buffer containing $25 \mathrm{mM}$ NaPIPES, $140 \mathrm{mM} \mathrm{NaCl}, 0.6 \%$ CHAPS, $0.14 \%$ PC, and a cocktail of protease inhibitors, and then boiled for 5-10 min in $1 \times$ SDS loading buffer. For Western blot of erythrocytes from KO mice and control littermates, cell lysates were prepared using RIPA lysis buffer (Beyotime) containing a cocktail of protease inhibitors (Roche ${ }^{24}$. The immunoprecipitated samples and cell lysates as loading controls were subjected to SDS-PAGE gels and electrophoresis separation. The separated proteins in the gel were transferred to PVDF membrane at $100 \mathrm{~V}$ for $2 \mathrm{~h}$. The membrane was blocked with 5\% blotting-grade blocker (Bio-rad) in TBST buffer (TBS buffer with $0.1 \%$ Tween-20) and incubated with the primary antibodies [anti-Piezo1 (1:1000), anti-GAPDH (Abcam, 1:3000) or anti- $\beta$-actin (Cell Signaling Technology, 1:3,000)] for overnight. After washing with the TBST buffer for 3 times, the membrane was incubated with the peroxidase-conjugated anti-rabbit IgG secondary antibody (CST, 1:10000) or anti-mouse IgG secondary antibody (Pierce, 1:20000) at room temperature for $1 \mathrm{~h}$, followed with wash and detection using the enhanced chemiluminescence (ECL) detection kit (Pierce). All uncropped images of blots are shown in Supplementary Fig. 4.

Measurement of body weight, heart weight, and tibia length. Mice were weighed and then sacrificed. Hearts were rapidly removed, trimmed to remove major blood vessels, sectioned, blotted, and then weighed. After the mice were euthanized, the tibias were dissected and the tibia lengths were measured.

Histological analysis. Excised hearts were quickly rinsed in the PBS buffer without $\mathrm{Ca}^{2+}$, and incubated in $4 \%$ PFA for at least $24 \mathrm{~h}$ at room temperature. Samples were dehydrated with ethanol, mounted in paraffin, and sectioned at $5 \mathrm{~mm}$ thickness. The sections were subjected to either H\&E staining or Masson's trichrome staining for visualizing the tissue architecture.
Immunocytochemistry. Heart paraffin sections were incubated at $60^{\circ} \mathrm{C}$ for $1 \mathrm{~h}$ and deparaffinized and rehydrated. For heat-induced antigen retrieval, the slides were boiled in a sodium citrate buffer for $10 \mathrm{~min}$ and then cooled down to room temperature for two times. After washing with distilled water for $5 \mathrm{~min}$, the sections were permeabilized with $0.2 \%$ Triton X-100 in PBST (PBS with $0.1 \%$ Tween 20) for $10 \mathrm{~min}$ and blocked with $5 \%$ donkey serum in PBST for $1 \mathrm{~h}$ at room temperature, then incubated with the rabbit anti-Flag antibody $(1: 500)$ at $4{ }^{\circ} \mathrm{C}$ overnight, subsequently washed with PBST for 3 times. The sections were incubated with the secondary antibody, Alexa Fluor-488 donkey anti-rabbit (1:500, Invitrogen) for $1 \mathrm{~h}$ at room temperature. After washing with PBST for 3 times, the sections were incubated with DAPI, then dried and mounted for confocal imaging.

For adult ventricular cardiomyocytes immunostaining, cells were isolated and plated on the laminin-coated coverslips and fixed with $4 \%$ PFA for 15 min, washed 3 times with PBS, and permeabilized with $0.2 \%$ Triton X-100 for $10 \mathrm{~min}$. Cells were blocked with 3\% bovine serum albumin (BSA) in TBST for $1 \mathrm{~h}$ at room temperature, then incubated with primary antibodies and secondary antibodies for $1 \mathrm{~h}$ at room temperature, respectively. Anti-Flag antibody (1:500, Sigma), antiSERCA2 (1:500, Thermofisher), anti-actinin (1:500, Sigma), anti-RyR2 (1:100, Thermofisher) were used. Secondary antibodies including Alexa Fluor 488 donkey anti-rabbit (1:500, Invitrogen), Alexa Fluor-594 donkey anti-rabbit (1:500, Invitrogen), Alexa Fluor 594 donkey-anti-mouse (1:500, Invitrogen) or Alexa Fluor 488 donkey-anti-mouse (1:500, Invitrogen) were used.

All the imaging procedures were performed on the Nikon Al confocal microscope (Nikon Instruments) with a $100 \times$ oil objective (N.A. $=1.49)$ using the $488 \mathrm{~nm}$ excitation wavelength and the $562 \mathrm{~nm}$ excitation wavelength. The images were analyzed using the Nikon NIS-Elements AR software. Co-localization of Piezo1 and SERCA2 or RyR2 either near the sarcolemma or inside the sarcoplasma of myocytes was calculated as Pearson's co-localization efficiency using the ImageJ software.

Fura-2 single cell $\mathrm{Ca}^{2+}$ imaging. Cardiomyocytes grown on the laminin-coated 8$\mathrm{mm}$ round glass coverslips were washed with the $1.8 \mathrm{mM} \mathrm{Ca}^{2+}$ Tyrode's solution (with BDM). And then incubated with $2.5 \mu \mathrm{M}$ Fura-2-AM (Life technologies) and $0.05 \%$ Pluronic F-127 (Life technologies) for $30 \mathrm{~min}$ at room temperature. The coverslip was mounted into an inverted Nikon-Tie microscopy equipped with a CoolSNAP CCD camera and Lambda XL light box (Sutter Instrument), and cells were selected for measurement of the 340/380 ratio with a $20 \times$ objective (N.A. $=$ 0.75) using the MetaFluor Fluorescence Ratio Imaging software (Molecular Device). $30 \mu \mathrm{M}$ Yodal was used for chemical activation of Piezol and $10 \mathrm{mM}$ caffeine for measuring SR $\mathrm{Ca}^{2+}$ content via activating RyR2.

Single cell $\mathbf{C a}^{2+}$ imaging by TIRF. Cardiomyocytes grown on the $35 \mathrm{~mm}$ glassbottom confocal dishes (Cellvis) were incubated with $2 \mu \mathrm{M}$ Fluo-4-AM (Life technologies) for $20 \mathrm{~min}$ at $37^{\circ} \mathrm{C}$ and an additional $10 \mathrm{~min}$ for de-esterification. And then scanned using a $488 \mathrm{~nm}$ laser in a TIRF microscopy (Nikon). Automated analysis of images was performed with Image J software installed the xySpark plugin $^{59}$.

$\mathrm{Ca}^{2+}$ spark and wave measurements. Cardiomyocytes were loaded with $2 \mu \mathrm{M}$ Fluo-4 AM for $20 \mathrm{~min}$ at $37^{\circ} \mathrm{C}$ and an additional $10 \mathrm{~min}$ for de-esterification ${ }^{44,60}$ Cells were plated on laminin-coated $35 \mathrm{~mm}$ glass-bottom confocal dishes and scanned using a $488 \mathrm{~nm}$ laser in a confocal line-scan mode. Automated analysis of line-scan images for $\mathrm{Ca}^{2+}$ sparks was performed using Image $\mathrm{J}$ software ${ }^{61}$ and Origin9.0. For low-concentration of caffeine response, adult cardiomyocytes were scanned for $120 \mathrm{~s}$ and $1 \mathrm{mM}$ caffeine were added into dish after $60 \mathrm{~s}$ record.

Cardiomyocyte attachment and stretch. All experiments were performed on the Nikon A1 confocal microscope (Nikon Instruments) with a $60 \times$ water objective (N.A. $=1.80)$ using the $488 \mathrm{~nm}$ excitation wavelength. The Stiff, a $25 \mu \mathrm{m}$ diameter glass micro-rod (WPI), was coated with MyoTak ${ }^{\mathrm{mm}}$ (WPI), which is a biocompatible cellular adhesive for attaching a single cardiomyocyte to research tools without damaging the cell. One glass micro-rod was connected to a high-sensitivity force transducer (WPI), and the other to a piezo-electric length controller (WPI) driven by a variable voltage output source. The glass-rods were positioned with motorized micromanipulators (WPI) and glued to the end of a single cardiomyocyte. The attached cardiomyocytes were given either an $8 \%$ or a $15 \%$ of axil stretch of the cell length. The Fluo-4 fluorescent signals before, during, and after the stretch stimulation were recorded by line-scan mode of confocal microscopy. The data were analyzed using the ImageJ software.

\section{$\mathbf{2}^{\prime}, \mathbf{7}^{\prime}$-dichlorofluorescein diacetate (DCF) measurement of reactive oxygen} species (ROS). Cardiomyocytes were loaded with $1 \mu \mathrm{M}$ DCFH-DA (Beyotime) for $20 \mathrm{~min}$ to measure $\mathrm{ROS}^{44}$. Non-fluorescent DCFH could be oxidized to fluorescent DCF. Detecting the fluorescence of DCF can quantify the level of ROS in myocytes. All experiments were performed on the Nikon A1 confocal microscope (Nikon Instruments) $100 \times$ oil objective (N.A. $=1.49)$ using the $488 \mathrm{~nm}$ excitation wavelength. A total of $30 \mu \mathrm{M}$ Yodal (Maybridge) was immediately added before camera shooting. For testing the blocking effect of Racl, DCF images were taken and compared before and after application of $50 \mu \mathrm{M}$ Racl inhibitor (APExBIO) 
together with $30 \mu \mathrm{M}$ Yoda1. For testing the blocking effect of NOX2, $3 \mu \mathrm{M}$ membrane permeable gp91ds-tat (Anaspec) were pre-incubated $30 \mathrm{~min}$ before the application of Yoda1, and the images were taken and compared before and after the application of Yoda1. Cells were imaged at low laser intensity and the $488 \mathrm{~nm}$ laser was shut off during the drug delivery process to ensure that the same position was taken twice without causing artifactual signal changes of DCF.

For measuring stretch-induced ROS, cardiomyocytes were loaded with $1 \mu \mathrm{M}$ DCF for $20 \mathrm{~min}$ as described above. The attached cardiomyocytes were given an $8 \%$ of axil stretch of the cell length and recorded DCF fluorescence intensity. DCF loaded cells were imaged using confocal line-scanning microscopy. As DCF can produce artifactual signal amplification upon continuous light exposure, cardiomyocytes were imaged at low laser intensity. Meanwhile, the attached cells were first recorded for the baseline fluorescence without stretch, then followed by stretch-induced fluorescence change. The baseline fluorescence was subtracted from the stretch-induced fluorescence change to avoid artifactual fluorescence change caused by illumination of DCF itself. Confocal line-scans were taken from the interior of the myocytes in the stretched region of the cells.

Echocardiographic analysis. Mice were anesthetized and the cardiac function was evaluated on conscious mice using a Vevo 2100 Ultrasound system. The images of long-axis view of left ventricle (LV), short-axis view of LV, and apical fourchamber view were obtained (Supplementary Table 1a-f).

Electrocardiogram (ECG) recoding. Mice were lightly anesthetized with isoflurane vapor $(0.5-1 \%)$ and $95 \% \mathrm{O}_{2}$. Anesthetized mice were placed on a pad, and subcutaneous needle electrodes were inserted into the right upper limb and hind limbs for continuous ECG recordings lasting at least 5 min after the heart rate became stabilized.

Human heart samples. The study was approved by the Ethics Committee of Fuwai Hospital and adhered to the Declaration of Helsinki. The study of the human heart samples was approved by the ethics committee of the institutional review board at Fuwai Hospital in Beijing. All experiments were complied with all relevant ethical regulations. All the patients who participated in the study provided written informed consent prior to sample collection and all other procedures and we have obtained consent to publish the information. The human sample information is shown in the Supplementary Data 1.

Data analysis. All data are shown as mean \pm standard error of mean (SEM). Statistical significance was evaluated using either unpaired or paired Student's t-test for analyzing two groups of samples, one-way or two-way ANOVA for analyzing multiple groups of samples.

\section{Data availability}

All relevant data are available from the corresponding author upon reasonable request. Source data are provided with this paper.

Received: 18 March 2020; Accepted: 19 January 2021; Published online: 08 February 2021

\section{References}

1. Friedrich, O., Wagner, S., Battle, A. R., Schurmann, S. \& Martinac, B. Mechano-regulation of the beating heart at the cellular

level-mechanosensitive channels in normal and diseased heart. Prog. Biophysics Mol. Biol. 110, 226-238 (2012).

2. von Anrep, G. On the part played by the suprarenals in the normal vascular reactions of the body. J. Physiol. 45, 307-317 (1912).

3. Patterson, S. W. \& Starling, E. H. On the mechanical factors which determine the output of the ventricles. J. Physiol. 48, 357-379 (1914).

4. Cingolani, H. E., Perez, N. G., Cingolani, O. H. \& Ennis, I. L. The Anrep effect: 100 years later. Am. J. Physiol. Heart Circulatory Physiol. 304, H175-H182 (2013).

5. Hibberd, M. G. \& Jewell, B. R. Calcium- and length-dependent force production in rat ventricular muscle. J. Physiol. 329, 527-540 (1982).

6. Allen, D. G. \& Kentish, J. C. The cellular basis of the length-tension relation in cardiac muscle. J. Mol. Cell. Cardiol. 17, 821-840 (1985).

7. Allen, D. G. \& Kurihara, S. The effects of muscle length on intracellular calcium transients in mammalian cardiac muscle. J. Physiol. 327, 79-94 (1982).

8. Kentish, J. C. \& Wrzosek, A. Changes in force and cytosolic Ca2+ concentration after length changes in isolated rat ventricular trabeculae. $J$. Physiol. 506, 431-444 (1998).

9. Ward, M. L. et al. Stretch-activated channels in the heart: contributions to length-dependence and to cardiomyopathy. Prog. biophysics Mol. Biol. 97, 232-249 (2008).
10. Hu, H. \& Sachs, F. Stretch-activated ion channels in the heart. J. Mol. Cell. Cardiol. 29, 1511-1523 (1997).

11. Bers, D. M. Calcium cycling and signaling in cardiac myocytes. Annu. Rev. Physiol. 70, 23-49 (2008)

12. Coste, B. et al. Piezo1 and Piezo2 are essential components of distinct mechanically activated cation channels. Science 330, 55-60 (2010).

13. Coste, B. et al. Piezo proteins are pore-forming subunits of mechanically activated channels. Nature 483, 176-181 (2012).

14. Ge, J. et al. Architecture of the mammalian mechanosensitive Piezol channel. Nature 527, 64-69 (2015).

15. Zhao, Q. et al. Ion permeation and mechanotransduction mechanisms of mechanosensitive piezo channels. Neuron 89, 1248-1263 (2016).

16. Murthy, S. E., Dubin, A. E. \& Patapoutian, A. Piezos thrive under pressure: mechanically activated ion channels in health and disease. Nat. Rev. Mol. cell Biol. 18, 771-783 (2017).

17. Douguet, D. \& Honore, E. Mammalian mechanoelectrical transduction: structure and function of force-gated ion channels. Cell 179, 340-354 (2019).

18. Xiao, B. Levering Mechanically Activated Piezo Channels for Potential Pharmacological Intervention. Annual Rev. Pharmacol. Toxicol., https://doi. org/10.1146/annurev-pharmtox-010919-023703 (2019).

19. Wu, J., Lewis, A. H. \& Grandl, J. Touch, tension, and transduction - the function and regulation of piezo ion channels. Trends Biochem. Sci., https:// doi.org/10.1016/j.tibs.2016.09.004 (2016).

20. Ranade, S. S. et al. Piezo1, a mechanically activated ion channel, is required for vascular development in mice. Proc. Natl Acad. Sci. USA 111, 10347-10352 (2014).

21. Li, J. et al. Piezo1 integration of vascular architecture with physiological force. Nature, https://doi.org/10.1038/nature13701 (2014).

22. Retailleau, K. et al. Piezol in smooth muscle cells is involved in hypertensiondependent arterial remodeling. Cell Rep. 13, 1161-1171 (2015).

23. Rode, B. et al. Piezol channels sense whole body physical activity to reset cardiovascular homeostasis and enhance performance. Nat. Commun. 8, 350 (2017).

24. Cahalan, S. M. et al. Piezol links mechanical forces to red blood cell volume. Elife 4, https://doi.org/10.7554/eLife.07370 (2015).

25. Peyronnet, R. et al. Piezo1-dependent stretch-activated channels are inhibited by Polycystin-2 in renal tubular epithelial cells. EMBO Rep. 14, 1143-1148 (2013).

26. Blythe, N. M. et al. Mechanically activated Piezol channels of cardiac fibroblasts stimulate p38 mitogen-activated protein kinase activity and interleukin-6 secretion. J. Biol. Chem. 294, 17395-17408 (2019).

27. Beech, D. J. \& Kalli, A. C. Force sensing by piezo channels in cardiovascular health and disease. Arteriosclerosis, thrombosis, Vasc. Biol. 39, 2228-2239 (2019).

28. Nonomura, K. et al. Mechanically activated ion channel PIEZO1 is required for lymphatic valve formation. Proc. Natl Acad. Sci. USA 115, 12817-12822 (2018).

29. Fotiou, E. et al. Novel mutations in PIEZO1 cause an autosomal recessive generalized lymphatic dysplasia with non-immune hydrops fetalis. Nat. Commun. 6, 8085 (2015).

30. Lukacs, V. et al. Impaired PIEZO1 function in patients with a novel autosomal recessive congenital lymphatic dysplasia. Nat. Commun. 6, 8329 (2015).

31. Choi, D. et al. Piezol incorporates mechanical force signals into the genetic program that governs lymphatic valve development and maintenance. JCI insight 4, https://doi.org/10.1172/jci.insight.125068 (2019).

32. Wang, S. et al. Endothelial cation channel PIEZO1 controls blood pressure by mediating flow-induced ATP release. J. Clin. Investig., https://doi.org/10.1172/ JCI87343 (2016).

33. Zeng, W. Z. et al. PIEZOs mediate neuronal sensing of blood pressure and the baroreceptor reflex. Science 362, 464-467 (2018).

34. Liang, J. et al. Stretch-activated channel Piezol is up-regulated in failure heart and cardiomyocyte stimulated by AngII. Am. J. Transl. Res. 9, 2945-2955 (2017).

35. Zhang, T., Chi, S., Jiang, F., Zhao, Q. \& Xiao, B. A protein interaction mechanism for suppressing the mechanosensitive Piezo channels. Nat. Commun. 8, 1797 (2017).

36. Brini, M. \& Carafoli, E. Calcium pumps in health and disease. Physiological Rev. 89, 1341-1378 (2009).

37. Cheng, H., Lederer, W. J. \& Cannell, M. B. Calcium sparks: elementary events underlying excitation-contraction coupling in heart muscle. Science 262, 740-744 (1993)

38. Cheng, H. \& Lederer, W. J. Calcium sparks. Physiological Rev. 88, 1491-1545 (2008).

39. Chen, J., Kubalak, S. W. \& Chien, K. R. Ventricular muscle-restricted targeting of the RXRalpha gene reveals a non-cell-autonomous requirement in cardiac chamber morphogenesis. Development 125, 1943-1949 (1998). 
40. Zhang, M., Wang, Y., Geng, J., Zhou, S. \& Xiao, B. Mechanically activated piezo channels mediate touch and suppress acute mechanical pain response in mice. Cell Rep. 26, 1419-1431 (2019). e1414.

41. Zeng, T., Bett, G. C. \& Sachs, F. Stretch-activated whole cell currents in adult rat cardiac myocytes. Am. J. Physiol. Heart Circulatory Physiol. 278, H548-H557 (2000).

42. Syeda, R. et al. Chemical activation of the mechanotransduction channel Piezo1. eLife 4, https://doi.org/10.7554/eLife.07369 (2015).

43. Iribe, G. et al. Axial stretch of rat single ventricular cardiomyocytes causes an acute and transient increase in $\mathrm{Ca}^{2+}$ spark rate. Circulation Res. 104, 787-795 (2009).

44. Prosser, B. L., Ward, C. W. \& Lederer, W. J. X-ROS signaling: rapid mechanochemo transduction in heart. Science 333, 1440-1445 (2011).

45. Bers, D. M. Cardiac sarcoplasmic reticulum calcium leak: basis and roles in cardiac dysfunction. Annu. Rev. Physiol. 76, 107-127 (2014).

46. Khairallah, R. J. et al. Microtubules underlie dysfunction in duchenne muscular dystrophy. Sci. Signal 5, ra56 (2012).

47. Sun, W. et al. The mechanosensitive Piezol channel is required for bone formation. eLife 8, https://doi.org/10.7554/eLife.47454 (2019).

48. Baba, S. et al. Flk1 $(+)$ cardiac stem/progenitor cells derived from embryonic stem cells improve cardiac function in a dilated cardiomyopathy mouse model. Cardiovascular Res. 76, 119-131 (2007).

49. Glennon, P. E. et al. Depletion of mitogen-activated protein kinase using an antisense oligodeoxynucleotide approach downregulates the phenylephrineinduced hypertrophic response in rat cardiac myocytes. Circulation Res. 78 954-961 (1996).

50. Zhao, Q. et al. Structure and mechanogating mechanism of the Piezol channel. Nature 554, 487-492 (2018)

51. Terentyev, D. et al. Redox modification of ryanodine receptors contributes to sarcoplasmic reticulum $\mathrm{Ca}^{2+}$ leak in chronic heart failure. Circulation Res. 103, 1466-1472 (2008).

52. Ward, C. W., Prosser, B. L. \& Lederer, W. J. Mechanical stretch-induced activation of ROS/RNS signaling in striated muscle. Antioxid. redox Signal. 20, 929-936 (2014).

53. Chen, X. et al. A Feedforward Mechanism Mediated by Mechanosensitive Ion Channel PIEZO1 and Tissue Mechanics Promotes Glioma Aggression. Neuron 100, 799-815 (2018). e797.

54. Luers, C. et al. Stretch-dependent modulation of $\left[\mathrm{Na}^{+}\right] \mathrm{i},\left[\mathrm{Ca}^{2+}\right] \mathrm{i}$, and $\mathrm{pHi}$ in rabbit myocardium-a mechanism for the slow force response. Cardiovascular Res. 68, 454-463 (2005).

55. Bae, C., Sachs, F. \& Gottlieb, P. A. Protonation of the human PIEZO1 ion channel stabilizes inactivation. J. Biol. Chem. 290, 5167-5173 (2015).

56. Ran, F. A. et al. Genome engineering using the CRISPR-Cas9 system. Nat. Protoc. 8, 2281-2308 (2013).

57. Ehler, E., Moore-Morris, T. \& Lange, S. Isolation and culture of neonatal mouse cardiomyocytes. J. Vis. Exp., https://doi.org/10.3791/50154 (2013).

58. Livak, K. J. \& Schmittgen, T. D. Analysis of relative gene expression data using real-time quantitative PCR and the 2(-Delta Delta C(T)) Method. Methods 25, 402-408 (2001).

59. Steele, E. M. \& Steele, D. S. Automated detection and analysis of $\mathrm{Ca}(2+)$ sparks in $\mathrm{x}-\mathrm{y}$ image stacks using a thresholding algorithm implemented within the open-source image analysis platform ImageJ. Biophys. J. 106, 566-576 (2014).

60. Guatimosim, S., Guatimosim, C. \& Song, L. S. Imaging calcium sparks in cardiac myocytes. Methods Mol. Biol. 689, 205-214 (2011).
61. Picht, E., Zima, A. V., Blatter, L. A. \& Bers, D. M. SparkMaster: automated calcium spark analysis with ImageJ. Am. J. Physiol. Cell Physiol. 293 C1073-C1081 (2007).

\section{Acknowledgements}

We thank Dr. Ardem Patapoutian for sharing the Piezol ${ }^{\mathrm{fl} / \mathrm{fl}}$ mice; Dr. Wayne Chen for critical discussion; Dr. Zai Chang and other stuff at the animal facility center of Tsinghua University for maintaining mice; Yalan Chen at the Imaging Core Facility, Technology Center for Protein Sciences at Tsinghua University for assistance with Nikon Al confocal microscopy; Dr. Qing Xu at the Core Facilities Center at the Capital Medical University for assisting in echocardiography and hemodynamic experiments. This work was supported by grants from the the National Key R\&D Program of China (2016YFA0500402) and the National Natural Science Foundation of China (31630090 and 31825014) to B.X.

\section{Author contributions}

F.J. carried out the bulk of the experiments and analyzed data; K.W. and M.Z. helped the initial characterization of the Piezol-TG mice; H.C. and S.W. provided technique help; K.Y. and Z.Z. provided the human heart samples; B.X. conceived and directed the study, analyzed data, and wrote the manuscript with help from all other authors.

\section{Competing interests}

The authors declare no competing interests.

\section{Additional information}

Supplementary information The online version contains supplementary material available at https://doi.org/10.1038/s41467-021-21178-4.

Correspondence and requests for materials should be addressed to B.X.

Peer review information Nature Communications thanks Jun Ren, Craig Simmons and the other, anonymous, reviewer(s) for their contribution to the peer review of this work. Peer reviewer reports are available.

Reprints and permission information is available at http://www.nature.com/reprints

Publisher's note Springer Nature remains neutral with regard to jurisdictional claims in published maps and institutional affiliations.

(c) (i) Open Access This article is licensed under a Creative Commons Attribution 4.0 International License, which permits use, sharing, adaptation, distribution and reproduction in any medium or format, as long as you give appropriate credit to the original author(s) and the source, provide a link to the Creative Commons license, and indicate if changes were made. The images or other third party material in this article are included in the article's Creative Commons license, unless indicated otherwise in a credit line to the material. If material is not included in the article's Creative Commons license and your intended use is not permitted by statutory regulation or exceeds the permitted use, you will need to obtain permission directly from the copyright holder. To view a copy of this license, visit http://creativecommons.org/ licenses/by/4.0/.

(c) The Author(s) 2021 